\title{
TAMING THE INCOMPUTABLE, RECONSTRUCTING THE NONCONSTRUCTIVE AND DECIDING THE UNDECIDABLE IN MATHEMATICAL ECONOMICS
}

K. Vela Velupillai 
The Discussion Paper series provides a means for circulating preliminary research results by staff of or visitors to the Department. Its purpose is to stimulate discussion prior to the publication of papers.

Requests for copies of Discussion Papers and address changes should be sent to:

Dott. Stefano Comino

Dipartimento di Economia

Università degli Studi

Via Inama 5

38100 TRENTO ITALIA 


\title{
Taming the Incomputable, Reconstructing the Nonconstructive and Deciding the Undecidable in Mathematical Economics*
}

\author{
K. Vela Velupillai \\ Department of Economics \\ University of Trento \\ Via Inama, 5 \\ 38100 Trento \\ Italy
}

Invited Paper, Presented at the Conference on:

Logic and Time: In Honour of Kurt Gödel

Rio de Janeiro, August, 27-29, 2007

Dedicated to the memory of J Barkley Rosser on the occasion of his centennial

September 27, 2007

\footnotetext{
${ }^{*}$ I first came across one of the many fundamental results of Chico Doria and Newton da Costa via two references in Ian Stewart's article on 'Deciding the Undecidable', in Nature, in 1991 ([93]). His references to two separate papers by da Costa and Doria sent me on a wild goose chase for several months. Eventually I managed to get the two papers ([18], [19]), written jointly, of course! Over the ensuing decade and a half, their important insights and pioneering work in many areas of dynamical systems theory and the foundations of mathematics has had a deep influence on my thought. My indebtedness to them is immense. Tom Boylan, Duncan Foley and Stefano Zambelli have shown the kind of critical support that makes it possible to choose an exotic research agenda (I have in mind a use of this word in the sense in which da Costa and Doria make 'exotic' assumptions in foundational work). Alas, I alone am responsible for the remaining errors and infelicities.
} 


\begin{abstract}
It is natural to claim, as I do in this paper, that the emergence of nonconstructivities in economics is entirely due to the formalization of economics by means of 'classical' mathematics. I have made similar claims for the emergence of uncomputabilities and undecidabilities in economics in earlier writings. Here, on the other hand, I want to suggest a way of confronting uncomputabilites, and remedying non-constructivities, in economics, and turning them into a positive force for modelling, for example, endogenous growth, as suggested by Stefano Zambelli ([107], [108]). In between, a case is made for economics to take seriously the kind of mathematical methodology fostered by Feynman and Dirac, in particular the way they developed the path integral and the $\delta$-function, respectively. A sketch of a 'research program' in mathematical economics, analogous to the way Gödel thought incompleteness and its perplexities should be interpreted and resolved, is also outlined in the concluding section.
\end{abstract}




\section{A Preamble}

"It is always right to be extremely suspicious when proofs in mathematics are taken with greater generality than is warranted by the known application of the proof. This is always a case of the mistake that sees general concepts and particular cases in mathematics. In set theory we meet this suspect generality at every step."

Wittgenstein: Philosophical Grammar ([104], p.467; italics added)

Solomon Feferman recently ([25], p.99) referred to '[Gödel's] stunning incompleteness results in 1931' (italics added). Robert Wolf, ([105], p. 151), in referring to the First Incompleteness Theorem and its proof was no less effusive: 'what Gödel did prove was .. a shattering revelation'. Just for the record, a precise and concise version of this theorem is as follows ([105], p.150):

Theorem 1 1. Theorem 2 Let $K$ be an axiomatizable extension of Peano Arithmetic. Then there is a sentence $Q$ of the language of $K$ such that:

(a) If $K$ is consistent, then $K \nvdash Q$, and

(b) If $K$ is $\omega$-consistent, then $K \nvdash \sim Q$, so $Q$ is independent of $K$

However, as in all careful expositions of the Incompleteness Theorems, Wolf's elegant book also pointed out the role played by the assumption of $\omega$-consistency in Gödel's proof. But unlike many other expositions - rigorous or (misleadingly) popular $^{1}$ - Wolf not only went on to clarify, with exemplary care, how Rosser, in $1936([75])^{2}$, weakened the assumption of $\omega$-consistency and replaced it with 'ordinary consistency'; but he also gave a crystal clear explanation of the difference in interpretation between a 'Gödel sentence' and a 'Rosser sentence':

"Whereas Gödel's sentence $[Q]$ expresses that 'There is no proof of me,' Rosser's sentence expresses the more intricate notion, 'For any proof of me, there is a proof of my negation with a smaller Gödel number.' This was a brilliant innovation on Rosser's part."

[105], p.152; italics added.

\footnotetext{
${ }^{1}$ An excellent exposition of the legions of misleading and incorrect assertions made by scores of mathematically illiterate attempts at making these theorems tell and do, more than they can or were intended to do, can be found in the fine recent book by the late Torkel Franzén. The review of this book by Gary Mar, done with touching sensitivity, is equally brilliant ([28],[54]). A melancholy example, on the other hand, of a misleading 'popular' exposition of 'Gödel's Theorems' is [31]. It escapes the sharp and perceptive analysis of Franzén, posibly simply because it was published in the same year!

${ }^{2}$ Franzen (op.cit, p.3; italics added) also observed:

"The property of $\omega$-consistency which Gödel assumed in his proof, is a stronger property than consistency and has a technical flavour, unlike the more readily understandable notion of consistency. However, the American logician J. Barkley Rosser showed in 1936 that Gödel's theorem could be strengthened so that only the assumption of plain consistency was needed to conclude that the system is incomplete."
} 
This conference is a celebration of Gödel and his deep visions and contributions to logic and time ${ }^{3}$. It so happens that this year marks the Rosser Centennial. I want, therefore, to pay homage also to Rosser ${ }^{4}$ - at least with a nod in this brief preamble - for having made the significance of the 'shattering' First Incompleteness Theorem, and its 'revelations', more readily comprehensible and slightly less technical. This laudable characteristic in Rosser, of being able to simplify over-general concepts and replace them with more down-to-earth ones, was also evident in at least two of his wonderfully pedagogical books: Logic and Simplified Independence Proofs ([77], [78]). In these two elegant books Rosser took a mathematically able novice, literally by the hand, and gently brought such a person to the frontiers of research in mathematical logic. The former is in that class of remarkable collection of pedagogically outstanding books written by the pioneers of logic and recursion theory, Kleene, Church, Davis and Rogers ([43], [16], [21], [74]) after Gödel and Turing ([99]); the latter was, for many years, in a class of its own.

But there are additional reasons, in the context of this paper, why I would like to invoke that classic Rosser 1936 paper. The best way to make my point would be to quote Rosser on the relevant (methodological) point:

"[A] modification is made in Gödel's proofs of his theorems ... . The modifications of the proofs make these theorems hold for a much more general class of logics. Then, by sacrificing some generality, it is proved that simple consistency implies the existence of undecidable propositions (a strengthening of Gödel's Satz VI ..) and that simple consistency implies the non-existence of an Entscheidungsverfahren ... . The class of logics for which these two results are proved is more general in some respects and less general in other respects than the class of logics for which Gödel's proof of Satz VI holds ..... ."

[75]; p. 87; italics added.

Rosser has deftly applied the classic trade-off that great mathematicians consciously make between weakening/strengthening conclusions and weakening/strengthening hypotheses, when facing a result - i.e., theorem - that is complex or over-general in some definable sense (a point made with characteristic force by Wittgenstein in the opening quote of this section). This is a

\footnotetext{
${ }^{3}$ No better, easily accessible, discussion of Gödel's philosophy of time can be found, in the published literature on his contributions to an interpretation of time in a world of Einstein, than in Palle Yourgrau's lucid writings (cf, for example, [106]).

${ }^{4} \mathrm{My}$ very first acquaintance with the name 'Rosser' occurred in my own initial attempt at learning Mathematical Logic using Willard Van Orman Quine's book, Mathematical Logic ([70]). Fortunately the cheap second hand edition I bought for myself, in the early 70 s was the 'Revised Edition'; it was also after I had read about Russell's Paradox, its discovery and the dismay it had caused Frege himself. This latter saga is, of course, well documented and part of the noble spirit of intellectual integrity. So, when I read in Quine's 'Preface to the Revised Edition' that 'the prime mover of the most important revision was Rosser' and that 'the revisions in [the revised edition had] affected half the pages', my curiosity, without the requisite maturity, was aroused. But it was not till over a quarter of a century later that I read Rosser seriously.
} 
standard method that constructive mathematicians routinely apply in their constant struggle to constructivise classical mathematical theorems ([52], [97]). A paradigmatic example will be discussed in the next section. Rosser's use of this methodology in a conscious and determined way to one of the great theorems of mathematical logic is, surely, to be applauded and, more importantly, to be used as a pedagogical devise in mathematical education.

Secondly, at a much more elementary level, again given the context of this paper - the backdrop of a computable economist - I would like to take another 'Rosserian' pedagogical example. Rosser did not only write 'advanced' texts in mathematical logic from an elementary standpoint (pace Felix Klein!). He also wrote an exemplary elementary text on teaching calculus with a pocket calculator, obviously in the days before the ubiquity of the personal computer ([79]). I actively sought out this book - partly with a curiosity as to how one of the pioneers of the $\lambda$-calculus and the joint architect of the fundamental Church-Rosser theorem would write a book on elementary calculus that might be consistent with the advanced work on defining functions procedurally. Given the experiences of reading his more advanced books, I was naturally curious to know how Rosser would approach the problem of teaching such a well-mined topic as elementary calculus - famously troublesome at the introductory level from the point of view of motivation. I need not have had the slightest of misgivings. The very first chapter begins as follows:

"Calculus is mainly a study of functions. ... For $f$ to be a function, $f$ must be a rule, a procedure, or mechanism which for a given $x$ (in the domain of $f$ ) prescribes a unique $y$, called $f(x)$.

Throughout much of calculus ..... the main question about some function $f$ is: 'Given a numerical value of $x$, what is the unique corresponding numerical value $y=f(x)$ ?'

Why should this be a problem? For $f$ to be a function, it must prescribe $y$, and uniquely so. But there are ways of prescribing $y$ that do not give much of a clue as to how to calculate a numerical approximation for its value."

[79], pp. 10-11; italics added.

I suspect the modern student could easily have been introduced to LISP quite immediately after the first lecture of such an elementary calculus course ${ }^{5}$, and, indeed, even to Church's Thesis. As a computable economist, familiar with the basics of the $\lambda$-calculus, I discern in this kind of definition, compared to the intrinsically non-numerical, non-effective ones given in standard texts ${ }^{6}$,

\footnotetext{
${ }^{5}$ If one remembers that Church was trying to define, with the $\lambda$-calculus, functions as rules of computation rather than as graphs of argument -value pairs, then, of course, everything becomes easier.

${ }^{6}$ For a thoroughly non-effective and muddled definition of function, given in the name of rigour, see the recent advanced text in mathematical economics by Ok ([64], pp.20-22). Even reputable texts, for e.g., [69], p. 10, [34], p. 60, and even the classic of its genre, CourantJohn, give definitions divorced from the notion of approximation, numerical evaluation and the procedures and mechanisms that will have to be devised to determine the 'unique value
} 
claiming to be underpinned by 'rigour' (without ever defining it), the umbilical chord linking computational procedures, approximation ${ }^{7}$ and estimation right at the very beginning of the long journey into the higher realms of analysis. Economics, in particular, is replete with 'ways of prescribing $y$ that do not give much of a clue as to how to calculate a numerical approximation for its value'. Most mathematical economists routinely invoke the kind of definition given, for example in [69], p.10 (italics in the original):

"A function $f$ from $\Re_{1}$ into $\Re_{1}$ is a relation in which no two ordered pairs have the same first element"

How on earth can such a definition train and hone the economist's intuitions in ways that could make the subject meaningfully quantitative? No wonder, then, that the economist seems always to think that theory is about proving existence theorems and, at a second stage, procedures have to be devised, often unconnected with the methods used in the proof of existence - say of an equilibrium - to compute an approximation to the equilibrium. Rosser, a pioneer of recursion theory and a pedagogical maestro, links us with the original meaning of the subject, as it was conceived by Newton and Leibniz, in such a way that computation, approximation and numerical meaning play their decisive roles from the outset.

But, of course, Rosser was a pioneer of recursion theory in its $\lambda$-calculus incarnation, where the Church-Rosser theorem was cardinal. To be able to define a function for an elementary calculus audience, in such a way that it links up to the deepest notions of computable functions, is an achievement that may have seemed natural and simple for someone who was there, at the beginning, with Church, and the creation of the $\lambda$-calculus.

For all of these reasons - and, in the context of this conference, for making the Gödel Incompleteness Theorems simpler and more perspicacious - I pay homage also to Rosser.

\section{Mathematical Foundations}

"Moreover, I think the Platonistic philosophy of mathematics that is currently claimed to justify set theory and mathematics more generally is thoroughly unsatisfactory and that some other philosophy grounded in inter-subjective human conceptions will have to be

of $y$ '. On the other hand, for an enlightened modern definition, entirely in line with Rosser's approach, with appropriate warnings when non-constructive reasonings are invoked, Estep's recent textbook is exemplary ([24]). The link calculus $\rightarrow$ functions $\rightarrow$ procedure $\rightarrow$ numerical approximation is reflected in Estep's innovative definitional statement that 'analysis means approximation and estimation' (ibid,p.1). I shall have the pleasure of returning to issues raised by Estep later in this paper.

${ }^{7}$ Not long after his celebrated work on replacing $\omega$-consistency with simple consistency, Rosser also derived his famous theorem on a rigorous lower bound for the prime number theorem ([76]). This is the kind of result a genuine analyst, interested in computation and approximation, would be expected to work on - but few derive. 
sought to explain the apparent objectivity of mathematics. Finally, I see no evidence for the practical need for new axioms to settle open arithmetical and finite combinatorial problems."

Solomon Feferman ${ }^{8}$ ([25], pp. 109-10; italics added)

The task I have set myself in this paper is to outline the nature and the explicitly acknowledged methodological aim of mathematical economics from the point of view of what I shall call Algorithmic Economics. I coined the name Computable Economics, about seventeen years ago, at a time when I was trying, together with my friend Stefano Zambelli, to reformalize the fundamental concepts of economic theory - what I have referred to as the closure of economic theory - on the basis of recursion theory ${ }^{9}$. I want, now, to study the current, orthodox, formalization of economic theory and the resulting mathematical economics - and the foundational justifications explicitly given for it - from the point of view of recursion theory and constructive mathematics. Hence Algorithmic Economics, of which Computable Economics is a proper subset ${ }^{10}$. To some extent the best way to tell this story would be to describe - eschewing, as much as possible, Whig tendencies - how economic theory was mathematised. A full development of what is outlined here will form, in the near future, the core of such a narrative. In the limited time and space allowed, this will have to be an outline.

Let me begin by circumscribing, for the purpose of this paper, mathematical economics to mean general equilibrium theory, game theory and the generally recognised and accepted mathematical core of newclassical macroeconomics: i.e., recursive macroeconomics.

I want to begin, by way of an introduction to the main themes of uncomputabilities, undecidabilities, unsolvabilities and nonconstructivities in economics, when economic theory is viewed as algorithmic economics, with a discussion of three famous and classic examples in physics and mathematics. These

\footnotetext{
${ }^{8}$ This particular pungent observation by the always stimulating Feferman was preceded by an equally punchy footnote, (ibid,p.109; first set of italics added):

"CH [the Continuum Hypothesis] is just the most prominent example of many set-theoretical statements that I consider to be inherently vague. Of course, one may reason confidently within set theory (e.g., in ZFC) about such statements as if they had a definite meaning."

${ }^{9}$ In the sense in which it was suggested by Arrow, [2], p.S398 (although I was already in the happy position of taking these 'next steps' before I was able to invoke Arrow's admonition in support):

"The next step in [economic] analysis ... is a more consistent assumption of computability in the formulation of economic hypotheses."

${ }^{10}$ In the same sense in which Linear Programming is a 'subset' of Integer Linear Programming; Classical Mathematics a 'subset' of Constructive Mathematics; and, most importantly, from an economic point of view, the way in which orthodox rationality is a proper subset of 'bounded' rationality. In the latter case, the usual view is the converse inclusion. I have explained in other writings why this 'usual view' is misleading and mistaken.
} 
examples, the Feynman Path Integral, the Dirac $\delta$-function and Bishop's Constructive Mathematics (henceforth: BCM), give me the fulcrum around which I shall base my discussion of issues in mathematical economics from the point of view of algorithmic economics.

I shall assume that readers know the mathematics of the Feynman Path Integral (method) and the Dirac $\delta$-function; explanations, definitions and the formal mathematics of both of them are easily available at any level of textbook exposition. To assume knowledge of $B C M$ in the same way may not be justified, but cannot be helped. The best I can do is to refer to Bishop's classic ([4]) and the fine summary in the exposition by Bridges and Richman ([11], where $B C M$ is referred to as $B I S H)$.

In his recent Cantor Medal Award lecture given at the meeting of the German Mathematical Society ([53]), Yu Manin observed, with precise eloquence (for one who had invoked a God who was an 'unpredictable poet' in the opening sentence $\left.^{11}\right)$, as follows (p.9; italics added):

"[R]equirements of quantum physics considerably heightened the degree of tolerance of mathematicians to the imprecise but highly stimulating theoretical discourse conducted by physicists. This led, in particular, to the emergence of Feynman's path integral as one of the most active areas of research in topology and algebraic geometry, even though the mathematical status of path integral is in no better shape than that of Riemann integral before 'Kepler's Stereometry of Wine Barrels [Stereometria Doliorum Vinariorum] ${ }^{12}$. "

${ }^{11}$ The opening quote of the lecture was from Tasic ([95]; italics added):

"God is no geometer, rather an unpredictable poet."

My perplexity here is whether the qualifying adjective is necessary; i.e., poets, by definition, are unpredictable. Therefore it would have been sufficient to characterise God simply as a poet!

${ }^{12}$ I cannot resist the temptation to quote from a recent paper by Segre [88], p.3; italics added):

"Let us briefly recall Feynman's way of arriving [at] his mathematically non rigorous but physically extraordinary path-integral formulation of Quantum Mechanics". ...

The basic steps are [four]:

4. A mathematically meaningless limit for $n \rightarrow+\infty$ (and hence $\epsilon \rightarrow 0$ ) in which the mathematically meaningless object $\lim _{n \rightarrow+\infty}\left(\frac{1}{2 \pi \epsilon}\right)^{\frac{n}{2}} \Pi_{k=1}^{n-1} d q_{k}$ is replaced with the mathematically meaningless functional measure $[d q(\bar{t})]$.

Such a derivation is [most] impressive for the complete lack of mathematical meaning of [the fourth step] as well as it is impressive for the geniality of the physical intuition underlying it, a geniality resulting in the physically extraordinary path-integral formulation of Quantum Mechanics, with no doubt one of the greatest achievements of $20^{t h}$ - century's Theoretical Physics"

Alas, no such felicitous example of mathematically meaningless derivation of an economically intuited great result can be found in $20^{t h}$ century mathematical economics. Why not? I conjecture - and shall try to make a case below - that it is because the mathematical economists have pawned their economic intuition (if ever they had one) to the broker dealing in classical mathematics and its axiomatic handmaiden, $Z F C$. 
Kepler, as we know, objected to the rule-of-thumb methods of wine merchants to estimate the liquid contents of a barrel of wine. I take it that Feynamn, here, is the wine merchant, and the rigorous mathematicians are to play the role of Kepler. In other words the Feynman path integral is to be interpreted as a 'rule-of-thumb' algorithm ${ }^{13}$. A clear understanding of Feynman's purpose in devising his 'algorithmic' path integral would clarify the role played by operational mathematics, physical intuition and a particular discipline provided by an epistemological question. The role of operational mathematics - by which I mean the classical mathematical tools, for example the kind one would learn in an advanced text on 'methods of mathematical physics', say of the classic Courant-Hilbert, [20], variety - and 'physical intuition', honed by quantum mechanical theory, is readily available in the technical literature and is fairly well known. But the epistemological aspect which added a particular discipline to Feynman's quest which led to the invention of the path integral is less well known. Feynman had been searching the literature - mathematical as well as mathematical physics - for earlier attempts that may have connected quantummechanical dynamics with Lagrangians or Hamiltonian principles. This is where the 'methodological discipline' entered decisively in the invention of the path integral, as told by Feynman himself ([27], p. viii; italics added):

"During some conversations with a visiting European physicist, Feynman learned of a paper in which Dirac had suggested that the exponential function of $i \epsilon$ times the lagrangian was analogous to a transformation function for the quantum-mechanical wave function in that the wave function at one moment could be related to the wave function at the next moment (a time interval $\epsilon$ later) by multiplying with such an exponential function.

The question that then arose was what Dirac had meant by the phrase 'analogous to,' and Feynman determined to find out whether or not it would be possible to substitute the phrase 'equal to.' A brief analysis showed that indeed this exponential function could be used in this manner directly. ...

However, in the application of this function it is necessary to carry out integrals over all space variables at every instant of time. ...

[T] he idea of 'integral over all paths' was developed as a way of both describing and evaluating the required integration over space coordinates. ....Actually, the path integral did not then provide, nor has it since provided, a truly satisfactory method of avoiding the divergence difficulties of quantum electrodynamics, but it has been found to be most useful in solving other problems in that field."

\footnotetext{
${ }^{13} \mathrm{~A}$ few days ago I asked my friend Chico Doria whether he knew of any recent success in taming the Feynman path integral axiomatically. His answer, as always illuminating, suggested the addition of the qualification 'algorithm' to 'rule-of-thumb', although my native behavioural economic intution would have naturally suggested 'rule-of-thumb procedure'. The point, however, is that the 'rule-of-thumb' qualification to 'algorithm', suggests the nonaxiomatizable nature of the Feynman path integral, which is my main point.
} 
It was the impetus provided by the epistemological ('analogous to') Dirac hint, backed up by a full command of the operational mathematics of the "methods of mathematical physics', and a search for whether there was an 'algorithmic' way of representing ('describing') and computing ('evaluating') the 'required integration' that led to the construction of the 'path integral' as we know it today. Can this 'algorithm' be encapsulated by an axiom system? Could it have been 'derived' by starting from an axiom system? The former question remains an open one; the latter is, in my opinion, a pointless exercise, precisely in the sense of the last sentence of Feferman's quote with which this section opened, except that Feferman could have added 'physics' to 'open arithmetical and finite combinatorial problems.' The point really is whether axiomatic issues played any role in the invention of the path integral; whether 'rigorously' justifiable mathematics is (was!) necessary for the way Feynman went about devising the path integral; whether such a straitjacket would not have hindered the development of one of the most versatile conceptual and computational tools of 20th century theoretical physics. Clearly, the answers to all of these questions are in the negative.

I do not know of any example in mathematical economics where such a systematic search for a mathematical solution to a foundational economic problem was attempted, let alone succeeded in devising a new algorithmic device. The mathematical economist bends and moulds the economic concepts to fit the concepts and methods of classical mathematics, in the first stage. At a second stage, (illegitimate) approximations are attempted to compute demonstrably uncomputable and nonconstructive entities. More on this later, but the issue of approximation and the role played by Dirac's intuition in the development of the path integral leads me to the issue of the Dirac $\delta$-function ${ }^{14}$.

This powerful concept and mathematical device, eminently successful as a hilfenkonstruktion in physics - both theoretically and computationally - was, as is well known, denounced by von Neumann himself for being 'improper', from a mathematical point of view; i.e., not a proper function in the rigorous (sic!) mathematical sense. We know now that it was mathematics that had to broaden

\footnotetext{
${ }^{14}$ There is no better summary of my methodological approach to the $\delta$-function than the one given by an eminent mathematician, with complete candour, Jacob Schwartz, [[86], p.21]:

"The sorry history of the $\delta$-function should teach us the pitfalls of rigor. Used repeatedly by Heaviside in the last century, used constantly and systematically by physicists since the 1920 's, this function remained for mathematicians a monstrosity and an amusing example of the physicists' naiveté - until it was realized that the $\delta$-function was not literally a function but a generalized function. It is not hard to surmise that this history will be repeated for many of the notions of mathematical physics which are currently regarded as mathematically questionable. The physicist rightly dreads precise argument, since an argument which is only convincing if precise loses all its force if the assumptions upon which it is based are slightly changed, while an argument which is convincing though imprecise may well be stable under small perturbations of its underlying axioms."
}

The mathematical economists, alas, do not subscribe to this eminently sensible approach, advocated with such eloquence by a formidable mathematician. 
its conceptual domain so as to encapsulate the $\delta$-function, rather than the other way $_{\text {about }}{ }^{15}$. The points I wish to emphasize here, as in the case of the path integral, are the roles played by approximation and computation in the saga that led to Dirac's development of the $\delta$-function and Schwartz's generalization of the notion of the function concept to encapsulate Dirac's intuitive use of it in a rigorous mathematical formalism. First, therefore, Schwartz's ex post rationale of the state of affairs ex ante (ibid, pp.217-8; italics added):

" $[\mathrm{P}]$ ysicists lived [prior to the discovery of 'distributions', in November, 1944] in a fantastic universe which they knew how to manipulate admirably, and almost faultlessly, without ever being able to justify anything. In this way they made great advances in theoretical physics. They deserved the same reproaches as Heaviside: their computations were insane by standards of mathematical rigour, but they gave absolutely correct results, so one could think a proper mathematical justification must exist. Almost no one looked for one, though. ....

...[I]t's a good thing that theoretical physicists do not wait for mathematical justification before going ahead with their theories."

There is not a hope in hell that the mathematical economists would allow economic theorists to go ahead with their theories unless they are first sanctioned by so-called rigorous mathematics, which essentially means classical mathematics + ZFC, underpinned by a Platonic philosophy. Keynes, supreme in his intuitions about the workings of a capitalist economy, has effectively been banished from the citadels of economic theory on the grounds of being an infidel with respect to mathematical justification of his conceptual framework. It is easy to show that a Keynesian formalized framework will have to go beyond classical mathematics for it to be encapsulated mathematically.

But this was an aside. To return to my theme of computation and approximation, I shall have to go back to Dirac himself, as I did in the case of Feynman. Dirac's underlying methodological and epistemological viewpoints disciplined the way he understood and developed the frontiers of physics, with great success it must be noted. Five principles can be discerned in his actual activity as a supremely original mathematical physicist: mathematical beauty, symmetry, meaning, intuition and approximation. Continuing with the Feynman, Schwartz theme of the irrelevance of mathematical rigour in the development of mathematical physics, it may be useful to recall Henry Margenau's 1933 reflection on 'Dirac vs. von Neumann' on method and philosophy of method:

"While Dirac presents his reasoning with admirable simplicity and allows himself to be guided at every step by physical intuition - refus-

\footnotetext{
${ }^{15}$ The beautiful and poignant autobiography of Laurent Schwartz, the man who discovered 'distributions', or 'generalized functions', to generalize the notion of functions gives a fulsome discussion of the road from the Heaviside operator calculus, its justification by Wiener and van der Pol, its modified use by Dirac and the eventual mathematical justification of the Dirac $\delta$-function by Schwartz's own discoveries. See, in particular, chapter VI of A Mathematician Grappling with his Century ([87]).
} 
ing at several places to be guided by the impediment of mathematical rigour - von Neumann goes at his problems equipped with the nicest of modern mathematical tools and analyses it to the satisfaction of those whose demands for logical completeness are most exacting."

[47], p.279; italics added.

Dirac had to reconcile the need for approximations in the natural sciences, particularly in fundamental physics, with his disciplining criterion of 'mathematical beauty'. Formulas, theories and theorems had to be 'beautiful', a notion on which he had definitive views and a belief that was a driving force in his pioneering work in theoretical physics. But whatever criterion of 'mathematical beauty' one used or invoked, it was not self-evident that any 'formula', particularly those derived experimentally, or any particular 'approximation' would satisfy any such criterion. He qualified the role of formulae by the view that any formula has meaning and the beauty could be found in the meaning; and only physical intuition, a mastery of the subject matter and an understanding of the kind of operational mathematics mentioned earlier and immense mental and disciplined experimental effort would give that . As for 'approximation':

"It seemed to me [around 1918] that if one worked with approximations there was an intolerable ugliness in one's work, and I very much wanted to preserve mathematical beauty. Well, the engineering training which I received did teach me to tolerate approximations, and I was able to see that even theories based on approximations could sometimes have a considerable amount of beauty in them .... I think if I had not had this engineering training, I should not have had any success with the kind of work that I did later on, because it was really necessary to get away from the point of view that one dealt only with results which could be deduced logically from known exact laws which one accepted, in which one had implicit faith."

[47], p. 280; italics added.

Well, mathematical economists have been doing everything possible not 'to get away from the point of view' from which Dirac absconded! More importantly, he sensed the need for approximation in computations and the need for computations in extracting intuitively natural physical laws from mathematically beautiful theorems, which then may well have to be represented as formulas, whose own inner beauty had to be understood from their meaning. Thus, the Dirac program ${ }^{16}$, 'officially' eschewing the useless shackles of mathematical rigour and an underpinning in axiomatizable mathematical systems, led

\footnotetext{
${ }^{16}$ Laurent Schwartz's discovery of 'Distributions' was directly inspired by the mathematical perplexity it had aroused in him during his University days ([87], p.218):

"I believe I heard of the Dirac function for the first time in my second year at the ENS. I remember .. that those formulas were so crazy from the mathematical point of view that there was simply no question of accepting them. It didn't even seem possible to conceive of a justification. These reflections date back to 1935 , and in 1944, nine years later, I discovered distributions.. The original
} 
to a fruitful development of theoretical physics, but also mathematics (and even mathematical logic), in the same sense in which it was pointed out by Manin on the Feynman path integral and by Schwartz on the Dirac $\delta$-function ${ }^{17}$.

I come, finally, to constructive mathematics. Most of what I have to say refers to Bishop's constructive mathematics (BCM. [4]), but much of my discussion can be rephrased to hold for any variety of constructive mathematics ([11]). I want to discuss the roles of the intermediate value theorem (IVT), the Bolzano-Weierstrass theorem, the Hahn-Banach theorem and the axiom of choice (in constructive mathematics), as a prelude to dissecting, in a cursory and preliminary way, the roles they play in Mathematical Economics. In addition, there is the question of the axiomatization of $B C M$ and the encapsulation of the notion of algorithm used in $B C M$ in the form of a 'thesis', analogous to the Church-Turing thesis in recursion theory. Let me begin with the intermediate value theorem, simply because it is what a beginning student of mathematical economics would have encountered in an elementary lecture on analysis for economists. This example will allow me to link up with the explicit strategy adopted by Rosser to replace the assumption of $\omega$-consistency with 'simple' consistency in Gödel's Theorem (see $\S 1$, above).

The intermediate value theorem, in a simple form, is as follows:

Theorem 3 Let $f: \Re \rightarrow \Re$ be a continuous function, from the closed interval [a,b] to $\Re$; and let: $\left\{\begin{array}{c}f(a)<0 \\ f(b)>0\end{array}\right.$. Then $\exists x \in \Re \& 3 a<x<b$, s.t $f(x)=0$.

To the best of my knowledge no elementary or even intermediate analysis textbook or monograph ever warns an unwary reader that this 'elementary theorem' does not hold constructively (nor, of course, why it does not hold constructively). Consider the following function $f_{\gamma}(x)$, depending on a parameter $\gamma \in \Re:$

$$
f_{\gamma}(x)=\left\{\begin{array}{c}
x-2 \text { if } x-2<\gamma \\
\gamma \text { if } x-4 \leq \gamma \leq x-2 ; \text {; the diagram below almost accurately } \\
x-4 \text { if } \gamma \leq x-4
\end{array}\right.
$$

depicts the behaviour of this function (where, above the 'thick' line $\gamma>0$; and below it, $\gamma<0)$ :

The zero of $f_{\gamma}$ cannot be determined, obviously, without additional knowledge whether $\gamma \leq 0$ or $\gamma \geq 0$. For, the zero of $f_{\gamma}$ changes from 4 to 2 , for any

reflections remained with me, and became part of the accumulated material .. which remained in a corner of my mind, only to explode suddenly the night I discovered distributions , in November, 1944."

\footnotetext{
${ }^{17}$ The generalization of the function concept, inspired by the needs of theoretical physics as envisaged by a supremely intuitively rigorous theoretical physicist, that resulted in 'Distributions', had the important 'computational' purpose of being able to work with functions that were always differentiable. After the Weierstrassian 'monstrosities' had been discovered, first by Takagi, in 1903, in an explicit example, (cf. [94]), of functions that were 'continuous everywhere but differntiable nowhere', there was a hiatus waiting to be filled: a hiatus that could have been filled either by re-interpreting the notion of function or generalizing it. The latter path resulted - but not because it was an exercise in 'gratuitous generalization' ([87], p. 211); but because it was motivated by the needs of a theoretical problem solver.
} 


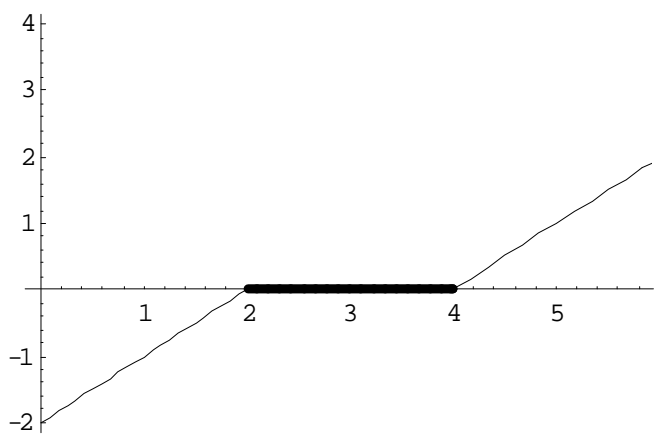

arbitrary change in the value of $\gamma$, from negative to a positive value. Therefore, the zero of $f_{\gamma}$ cannot be determined with any preassigned, desired, degree of accuracy. What would be the constructivist's strategy? The constructivist's standard method, when confronted with a 'classical' non-constructive theorem is to either weaken the conclusion or strengthen the hypothesis (see [52], p.274 and [98], p.23). The attentive reader may now note the similarity of this 'constructivising' strategy with that adopted by Rosser when replacing $\omega$-consistency with 'simple' consistency in Gödel's Theorem. To understand, for example how to weaken the conclusion, it is necessary to understand the non-constructive content of the original 'classical' theorem: the proof is by contradiction, assuming, first, that no point of $f$ lies on the axis and deriving a contradiction. The proof, by itself, says nothing about how to find the point on the curve for which $f(x)=0$. This is standard practice in existence proofs in 'classical' mathematics and, a fortiori, in mathematical economics.

Now armed with this knowledge, the constructivist can proceed to 'weaken the conclusion', which is done by observing that it is possible to determine constructively, $\forall \varepsilon>0$, a $x \in \Re \& a<x<b$ s.t $|f(x)|<\varepsilon$. A precisely formal constructivist $I V T$ :

Theorem 4 Let $f: \Re \rightarrow \Re$ be a continuous function, from $[a, b] \in \Re$ to $\Re$; and let: $\left\{\begin{array}{l}f(a)<0 \\ f(b)>0\end{array}\right.$; then, $\forall \varepsilon>0, \exists x \in \Re, a<x<b$, s.t $|f(x)|<\varepsilon$.

The constructivst's strengthening of the hypothesis results in a second constructive $I V T$ which is precisely what is needed to prove, constructively, that the reals are algebraically closed. One way to strengthen the hypothesis is to assume that $f(x)$ is a polynomial. It can be seen, then, that in the first case the constructivist obtains an approximation to the value of $x$ which determines a zero of the function; in the second case, the constructivist first approximates the given function (in this case by a polynomial) and then obtains an exact value of $x$ which determines $f(x)=0$. Either way, an approximation is precisely determined. The ubiquity of approximation in computation in the context of analysis is the reason for Estep to ask a pertinent question, on the very first page of his excellent intermediate level analysis text, and answer it as follows: 
"What is analysis? .. To 'applied' mathematicians, analysis means approximation and estimation. .... .

To 'pure' mathematicians, ..., analysis is the study of the limiting behaviour of infinite processes. ...

But these two viewpoints of analysis describe the same activity. Approximation and estimation imply some notion of limit, that is the possibility of obtaining full accuracy as the limit of the approximation process. On the other hand, the concept of a limit also implies an approximation that can be made as accurate as desired."

[24], pp. 1-2; italics added.

Of course, the 'pure mathematician' in the above definition is a constructive analyst, for, if not, the last sentence cannot make sense. This is what is highlighted by the proof of the 'elementary' IVT, which is devoid of any and all numerical content.

I shall, for reasons of space and time, be relatively brief about the BolzanoWeierstrass theorem, the Hahn-Banach theorem and the axiom of choice.

As for the Bolzano-Weierstrass theorem, the elementary calculus lesson will not always tell you that something like it underpins even the IVT. To get a flavour of this connection I refer the reader to Estep's (op.cit) definition of a Bisection Algorithm, it's role in what he calls 'Bolzano's theorem' and the IVT as a consequence of the two ([24], chapters, $10 \& 13$, esp. pp. 129-33 \& 16870). The main point is that the Bolzano-Weierstrass theorem is proved using a countable infinity of undecidable disjunctions and is not constructifiable.

The lesson from the way the IVT was modified to yield two constructive $I V T$ s is important in the constructivist's enterprise, as I indicated above. Thus, an exercise similar to the way the constructivist weakened the conclusion and strengthened the hypothesis to yield an approximately exact solution can, of course, be done for the Hahn-Banach theorem (cf. for example, [4], pp. 262-3; but also [12], §5.3). One loses the exact preservation of numerically meaningless 'classical' norms in the constructive version; but gains, as in the case of the $I T V$, numerical meaning and exact computational content in the constructive version.

The Hahn-Banach theorem, in other words, has a constructive version, but only on subspaces of separable normed spaces. The standard, 'classical' version, valid on nonseparable normed spaces depends on Zorn's Lemma which is, of course, equivalent to the axiom of choice, and is therefore, non-constructive ${ }^{18}$.

Schechter's perceptive comment on the constructive Hahn-Banach theorem is precisely the point I was making with my extensive discussion of the constructivising of the $I V T$ (ibid, p. 135; italics in original; emphasis added).:

\footnotetext{
${ }^{18}$ This is not a strictly accurate statement, although this is the way many advanced books on functional analysis tend to present the Hahn-Banach theorem. For a reasonably accessible discussion of the precise dependency of the Hahn-Banach theorem on the kind of axiom of choice (i.e., whether countable axiom of choice or the axiom of dependent choice), see [62]. For an even better and fuller discussion of the Hahn-Banach theorem, both from 'classical' and a constructive points of view, Schechter's encyclopaedic treatise is unbeatable ([84]).
} 
"[O]ne of the fundamental theorems of classical functional analysis is the Hahn-Banach Theorem; ... some versions assert the existence of a certain type of linear functional on a normed space $X$. The theorem is inherently nonconstructive, but a constructive proof can be given for a variant involving normed spaces $X$ that are separable - i.e., normed spaces that have a countable dense subset. Little is lost in restricting one's attention to separable spaces ${ }^{19}$, for in applied math most or all normed spaces of interest are separable. The constructive version of the Hahn-Banach Theorem is more complicated, but it has the advantage that it actually finds the linear functional in question."

At an elementary level, the same applies to the constructive versions of the IVT

So, one may be excused for wondering, why economists rely on the 'classical' versions of these theorems? They are devoid of numerical meaning and computational content. Why go through the rigmarole of first formalising in terms of numerically meaningless and computationally invalid concepts to then seek impossible and intractable approximations to determine uncomputable equilibria, undecidably efficient allocations, and so on?

These three theorems and the axiom of choice ${ }^{20}$ are the pillars of the citadel that is mathematical economics. They are all of them, without exception, completely devoid of numerical meaning (in the sense of Bishop - see Preface and Chapter 1 of [4] and [5]) and (hence) algorithmic content. The citadel is replete with deceptive claims for computable general equilibrium theory, recursive competitive equilibria, computationally efficient decentralized equilibria, and so on. These claims and assertions are to be taken with a barrel of salt. None of them are true because they rely, one way or the other, on one or more of the above three theorems and the axiom of choice.

In other words, the citadel is built on the sands of 'classical' mathematics and founded on ZFC. This is the reason for the ubiquity of uncomputabilities, undecidabilities, unsolvabilities and unconstructivities in mathematical economics. Mathematical economics, as it is today - in content and form - cannot be algorithmic economics. Hence, there is no sense in which there could have arisen analogues of a Feynman path integral or a Dirac $\delta$-function, or the kind of research strategies implemented by these two imaginative giants of 20th Century theoretical physics. Every celebrated theorem in mathematical economics, from Arrow's Impossibility Theorem, via the Nash and Arrow-Debreu equilibria, the two fundamental theorems of welfare economics, to macroeconomic recursive

\footnotetext{
${ }^{19}$ However, it must be remembered that Ishihara, [36], has shown the constructive validity of the Hahn-Banach theorem also for uniformly convex spaces.

${ }^{20}$ Incidentally, that famous 'bane' of the axiom of choice, the 'Banach-Tarski Paradox' has recently been shown to be implied by the Hahn-Banach Theorem (see [65]). Not with a bit of my tongue in my cheek, I have been wondering whether I should not try to 'prove' that the Banach-Tarski Paradox is implied by the second fundamental theorem of welfare economics. The traditional 'folk' assumption that 'there is no such thing as a free lunch' can then be ignored forever.
} 
competitive equilibria, is plagued by the infelicities common to one or more of the above theorems. Hence every one of them is rich in uncomputable, nonconstructive, undecidable and unsolvable content. A sketch of how to unearth them, based upon the discussion above, is outlined below.

Not very recently, Donald Knuth wondered about the difference between 'Algorithmic Thinking and Mathematical Thinking' ([45]). An 'experiment' he carried out, in trying to clarify the 'difference' (between the two kinds of 'thinking', if there was one), gave an illuminating result for Bishop's Foundations of Constructive Analysis ([4]):

"The last book I chose to sample turned out to be the most interesting of all from the standpoint of my quest: it was Errett Bishop's Foundations of Constructive Mathematics ${ }^{21}$, a book that I had heard about but never before read. The interesting thing about this book is that it reads essentially like ordinary mathematics, yet it is entirely algorithmic in nature, if you look between the lines."

[45], p.177; last set of italics italics added.

The problem with modern mathematical economic scholarship is that no one reads 'between the lines' any more, especially in books like Bishop's.

\section{Mathematical Economics}

"What relevance, if any, might constructive mathematics have for economic theory? There are several answers to such a question. For example, there is the generic response that the use of constructive methods reveals distinctions between computability and non-computability that are obscured, often totally, by classical 'existential' methods."

Douglas Bridges ([10], p.7; italics added).

My own research, since about 30 years ago has been on trying to understand the relevance of recursion theory and its applied branches ${ }^{22}$ for economic theory. In particular, the investigations of the computable, solvable and decidable implications of a more systematic assumption of computability in core areas of general equilibrium theory, game theory and macroeconomics. In much of this work I was working, consciously, within the discipline of the Church-Turing Thesis and what I came to call the Kolmogorov-Chaitin-Solomonoff-Martin-Löf Thesis in applied recursion theory. More recently I have felt the need to broaden the notion of algorithm I had been using in my investigation to include constructive mathematics - in particular, Bishop-style constructive analysis. That the Bishop-style

\footnotetext{
${ }^{21}$ A rare slip on Knuth's part! The word should have been 'analysis', not 'mathematics', in the title of Bishop's book.

${ }^{22}$ By the 'applied branches' of recursion theory I mean: computational, algorithmic and stochastic complexity theories, in the sense that these fields are based on the foundations of (classical) recursion theory.
} 
constructivists refuse to circumscribe their notion of algorithm formally as, for example, recursion theorists do, by accepting the Church-Turing Thesis, is more in line with my current Dirac/Feynman-inspired vision (see above) of the role of mathematics in the applied sciences. Moreover, in recent years, in lectures and in a few of my writings I expressed disquiet at the prevailing practice, in mathematical economics circles, of what seemed to be a tacit acceptance of the belief that the computable functions were a subset of a larger class of more generally defined and routinely accepted set of functions ${ }^{23}$. Finally, the fact that non-constructive existence proofs are routinely developed in recursion theoretic frameworks has also troubled my epistemological antennae. Fred Richman's recent perceptive remark encapsulates much of my above disquiet, particularly in the context of mathematical economics ${ }^{24}$ :

"My final example of a nonconstructive existence proof comes from the theory of computable functions. That theory was developed to clarify the idea of what it means for a function to be computable. This is not an idea that would occur to a constructivist, for whom computability is part of the intuitive idea of a function: if there exists $y$ such that $f(x)=y$, then we must be able to construct that $y$ if we are given $x$. A constructivist can certainly entertain the idea of looking at a restricted class of functions that are computed in a special way, but would be unlikely to call that class 'the computable functions'. "

[72], pp. 306-7; italics added.

\footnotetext{
${ }^{23}$ Over the years I have had fruitful discussions with my friend Francesco Luna on these matters. I stated, earlier in the paper, that I consider Classical Mathematics a particular subset of Constructive Mathematics; also, in an economic theoretic context, that bounded rationality is the more general notion and not the orthodox definition of rationality. The analogy, again as mentioned earlier in this paper (and in many of my various writings on 'computable economics'), is with the sense in which linear programming is a special case of integer linear programming. The former, by the way, is defined and studied over $\Re$; the latter, usually, over $\mathbb{Z}$ (sometimes also over $\mathbb{Q}$ ).

${ }^{24}$ The review by Ray Mines of [6] expresses these disquieting sentiments quite clearly ([56], pp. 162-3; italics added):

"Many people have expressed the view that there should be close ties between the theory of algorithms [i.e., computability theory] and constructive mathematics. Perhaps because algorithms are innately constructive. ... Times have changed. Mike Fellow[s] has used Wagoner's [sic!] conjecture to show that there are polynomial time algorithms for solving certain problems in graph theory. His methods are highly nonconstructive. In fact the existence of these algorithms is probably independent of $\mathrm{ZF}$. So, there are now pure existence proofs for computer programs that solve real problems, and no hope of ever programming them."
}

I think Mines is referring to Wagner's conjecture (not 'Wagoner'), [102], and to the result in [26], Theorem B, p. 728:

Any set of finite graphs contains only a finite number of minor-minimal elements.

A graph $H$ is called a minor of a graph $G$, if a graph isomorphic to $H$ can be obtained from a subgraph of $G$ be contracting edges. 
No wonder, then, that mathematical logicians who have been trying to find a formal system to encapsulate $B C M$ have had the same kind of difficulties as those who have attempted similar encapsulations of the Feynman path integral!

Against the backdrop of this brief preamble, I can now state that the mathematical 'crown jewels" ${ }^{25}$ in the citadel that is mathematical economics - general equilibrium theory and game theory - are the contraction mapping theorem, fix point theorems (I shall only consider the Banach Contraction Mapping theorem and the Brouwer and Schauder fix point theorems) and the Hahn-Banach (and associated separating hyperplane) theorems and, for mathematical macroeconomics, the (mathematically unfortunate and inappropriately named trio of ) recursive tools ${ }^{26}$ : dynamic programming $(D P)$, Kalman Filtering $(K F)$ and Markov Decision Processes $(M D P)$. In the former, equilibrium existence and their computations and the two fundamental theorems of welfare economics are the jewels; in the latter, the structure and computation of a recursive computational equilibrium (see, [17], Chapter 1 and [49], Chapter 12) is fast becoming the jewel. The existence proofs utilise the contraction mapping theorem, one or another fix point theorem, but overwhelmingly often it is the Brouwer Fix Point Theorem. Computation of the nonconstructively proved equilibrium is by means of various alleged 'algorithms', mathematically underpinned by Uzawa's 'equivalence theorem' ([92], Chapter, 11; [100]) The formalization and proof of the two fundamental theorems of welfare economics depend entirely on the Hahn-Banach theorem. The computation of a recursive computational equilibrium by the rational agent modelled as a signal processor is achieved by using the macroeconomic crown jewels: DP, KF and MDP.

It will be obvious for anyone familiar with the literature cited in the previous section, and the discussion of them in it, that these crown jewels carry with them intrinsic uncomputabilities, undecidabilities, unsolvabilities and nonconstructivities. The task of the following subsections is to be slightly more explicit about these blemishes in the crown jewels. I expect the discerning reader to notice that I am emphasizing blemishes in the jewels; not on where the crown sits, i.e., economic theory ${ }^{27}$.

\footnotetext{
${ }^{25}$ In analogy with the metaphor suggested in [62], p. 193 (italics added):

"In its elegance and power, the Hahn-Banach theorem is a favorite of almost every analyst. Some of its sobriquets include The Analyst's Form of the Axiom of Choice and The Crown Jewel of Functional Analysis. Its principal formulations are as a dominated extension theorem and as a separation theorem."
}

It is as a 'separation theorem' that it plays the 'crown jewel' role in economic theory.

${ }^{26}$ Hence Newclassical Macroeconomics is now referred to as Recursive Macroeconomics ([?]).

${ }^{27} \mathrm{On}$ the other hand, having been brought up immersed in the Bhaghawat Ghita, I am also aware that the headgear is considered part of the head of the wearer. In one of the most poignant events in the great battles between the Pandavas and the Kaurawas, the divine arrow fired by Karnan, a half-brother to his opponent Arjunan at whom the divine arrow was aimed, fulfilled its mission by piercing the crown worn by the latter. Thus, the arrow's divine mission was fulfilled; and Krishna, as Charioteer, succeeded in saving Arjunan by interpreting the headgear as part of the head on which the crown that was pierced sat. In finding blemishes in the jewels in the crown I may, as a cultural Hindu, be trying to pierce the head, too. I don't know. I will never know. 
My conviction, after all these years of struggling with an understanding of the non-algorithmic content of mathematical economics, is that the teleological element in mathematical economics is entirely attributable to the kind of mathematics that is used in its formalization. Years ago, reading the following profound observation by Bishop led me along a path towards an understanding of these teleological underpinnings and their sources in classical, non-algorithmic, non-intuitive, mathematics:

"The classical concept of a set as a collection of objects from some pre-existent universe is clearly inappropriate constructively. Constructive mathematics does not postulate a pre-existent universe, with objects lying around waiting to be collected and grouped into sets, like shells on a beach. The entities of constructive mathematics are called into being by the constructing intelligence. From this point of view, the very question 'What is a set?' is suspect. Rather we should ask the question, 'What must one do to construct a set?'. When the question is posed this way, the answer is not hard to find."

Errett Bishop: Schizophrenia in Contemporary Mathematics ([5], p.11; italics added).

Lurking behind almost all of these non-algorithmic proofs lies the spectre of the Axiom of Choice. Even in the simplest dynamic problem of the proof of existence of a solution to the classic Initial Value Problem of Ordinary Differential Equations (IVPs of $O D E \mathrm{~s}$ ), this axiom rears its head as a spectre right on the ground floor, in every sense of the word. Long before Zermelo gave content to the axiom in the form we all know and use it today, in fact precisely fourteen years before ${ }^{28}$, Peano had perceptively realized its potential ubiquity and immediately rejected it in one of his earliest papers on the IVP problem for ODEs. He knew what its non-constructive philosophical implications were and where it reared its head in the IVP problem for ODEs (cf., [57], §.1.8). His first objection, and probably the first formal recognition of the role played by the (implicit) assumption of the axiom of choice in such questions, to any appeal to this axiom, came in his pioneering paper on the role of the Lipschitz condition

\footnotetext{
${ }^{28}$ Peano referred to the objection he had earlier made to any appeal to what subsequently came to be called the axiom of choice in his 1906 paper on the Cantor-Bernsteim Theorem ([67], pp. 207-8; italics in original) as follows:

"Zermelo, in proving a proposition about well-ordered classes, adopted and stated the principle 'that even for an infinite totality of sets there are always mappings that associate with every set one of its elements .... . This logical principle cannot, to be sure, be reduced to a still simpler one, but it is applied without hesitation everywhere in mathematical deduction'.

This principle means that we may arbitrarily choose an infinite number of elements. This assumption, which occurs in several books, was already considered by me in the year 1890, in Math. Ann., 37, p. 210: 'one may not apply an infinite number of times an arbitrary law according to which to a class $a$ is made to correspond an individual of that class ....'
} 
in guaranteeing uniqueness of solutions for the IVP problem for ODE (cf. [35], p. 66 , footnote). Peano noted, with great perspicacity:

"[S]ince one cannot apply infinitely many times an arbitrary rule by which one assigns to a class $\mathrm{A}$ an individual of this class, a determinate rule is stated here, by which, under suitable hypotheses, one assigns to each class A a member of this class."

[66], p.210, quoted in: [57], p76; italics in the original ${ }^{29}$.

However, Peano's younger Italian colleagues were less scrupulous about appealing to, or implicitly using, the axiom of choice. Thus Giulio Ascoli had already in $1884^{30}$, ([3]) introduced the concept of equicontinuity, which now underpins the Ascoli-Arzela theorem, a theorem routinely used as a criterion for compactness $^{31}$, quite commonly in mathematical economics. Economists who appeal to this theorem, or even simply to equicontinuity, do not normally bother to investigate its non-constructive foundations - as little as they investigate the simpler Heine-Borel theorem for computability. I'll have something to say about these things in the context of specific economic examples in the next sub-section.

\subsection{Understanding Non-constructivity in Economics}

"Assuming that [a family $\Im$ of functions] is uniformly bounded and equicontinuous, it is sufficient [to show the existence of solutions to an integral equation] to show that it [ $\Im$ ] contains a uniformly convergent subsequence. The proof ..... appears to be constructive. However, each step requires deciding whether a given region contains the graphs of an infinite number of functions, something that cannot be checked by a computer.

[24], p. 589; italics added.

I shall begin with a discussion of fix point type models and their nonconstructive (and, occasionally, also non-computable) underpinnings in the first

\footnotetext{
${ }^{29}$ The original by Peano, in French, is ([66], p. 210; italics in original):

"Mais comme on ne peut pas appliquer une infinité de fois une loi arbitraire avec laquelle à une classe $a$ on fait corespondre un individu de cette class, on a formé, ici une loi déterminée avec laquelle à chaque classe $a$, sous des hypothèses convenables, on fai correspondre, un individu de cette classe."

${ }^{30}$ In the pseudo-rigorous [64] (footnote, 36, p. 263, a pretentious notein itself), the author is even sloppy in getting such a simple thing as this date right.

${ }^{31}$ I have never seen, in any mathematical economics text, at any level of sophistication, an appreciation of the non-computable and non-constructive contents of the Heine-Borel theorem, the Bolzano-Weierstrass theorem, the assumption of compactness, completeness and equicontinuity and many other routinely used mathematical concepts. These theorems and concepts are routinely used and appealed to by every kind of mathematical economist, without exception. Personally I find this an unmitigated disaster for anyone seriously interested in making economics a meaningfully numerical and quantitative subject.
} 
subsection. I shall then go on, in the second sub-section, to discuss the nonconstructive content and axiom of choice implications of the Hahn-Banach theorem.

\subsubsection{Contractions and Fix Points}

"[T]he famous contraction mapping theorem [is the one] by which Banach transformed a 'folk-technique' into a theorem."

Körner, [46], p. 287.

But in this wonderful and entertainingly clear advanced 'Companion to Analysis', with a characteristically paradoxical title ${ }^{32}$, Körner does not warn the unwary reader that 'folk-techniques', converted to theorems relying on nonconstructive assumptions, lose their 'folkiness'!

There is a whole chapter titled Fixed-Point Arguments in ([50], Chapter 17), in what has become the standard textbook for the mathematics of macroeconomics. Three fix point results are presented and applied in macroeconomic contexts: the contraction mapping theorem, the Brouwer fix point theorem and the Schauder fix point theorem. They are presented without the slightest comment on their algorithmic content, as if just the formalization of some canonical economic model - such as an overlapping generation model (OLG) - with the requisite properties that makes it possible to apply one or the other of these theorems makes them numerically and computationally ${ }^{33}$ meaningful.

In all three of the applications the problem, derived from a standard $O L G$ model, the task is to prove the existence of solutions, $f^{*}(x)$, ([50], chapter 17, p. 507), where: $x \in[a, b]=X \subset \Re_{++}$:

$$
f^{*}(x)=\int \phi\left[x^{\prime} \zeta^{-1}\left(f\left(x^{\prime}\right)\right)\right] \pi\left(x, d x^{\prime}\right), \forall x \in X
$$

where:

$\zeta:[0, L) \rightarrow \Re_{+}$

$\phi: \Re_{+} \rightarrow \Re_{+}$

$\pi: X \times \Theta \rightarrow[0,1]$, where $\Theta:$ the Borel subsets of $X$.

To study the existence of solutions $f^{*}(x)$ to $(1)$, the following operator $T$ on functions $f: X \rightarrow \Re_{++}$is defined (ibid, p.507) as:

$$
(T f)(x)=\int \phi\left[x^{\prime} \zeta^{-1} f\left(x^{\prime}\right)\right] \pi\left(x, d x^{\prime}\right)
$$

Obviously, $f^{*}(x)$ satisfies (1) iff $f$ it is a fixed point of $T$. For the rest, it is simply a case of making sure the OLG model satisfies the appropriate hypotheses

\footnotetext{
${ }^{32}$ The subtitle of $A$ Companion to Analysis is: 'A Second First and First Second Course in Analysis'!

${ }^{33}$ Although there are no lack of claims on 'computational' feasibilities, always using the word inappropriately (for example, [50], p.529: 'This corollary provides a useful computational check .....'). Nothing of the sort - at least if by 'computational' one means the rigorous concept underpinned by either constructive or computable analysis.
} 
to make it possible to apply the Contraction Mapping Theorem, the Brouwer Fix Point Theorem or the Schauder Fix Point Theorem. Not a single comment or reservation is made about the hypotheses so as to make any connection with numerical meaning or computational content.

The key mathematical hypothesis in the (Banach) contraction mapping theorem is on the structure of the domain: that it is a complete metric space. This assumption is made as if it is numerically and computationally innocuous. But it is not. Let me proceed systematically. First, what is completeness? ? $^{34}$

Definition 5 An ordered field is said to be complete if it obeys the monotone sequence property.

What, then, is the monotone sequence property?

Definition 6 Let $F$ be an ordered field. Then $F$ is said to have the monotone sequence property if every monotone increasing sequence bounded above converges $^{35}$.

Now, consider the following result, due to Specker ([90]), from Computable Analysis ([103]:

Theorem $7 \exists$ an increasing and bounded sequence of computable sequence of rational numbers whose limit is a non-computable real number.

What kind of numbers can Lucas, Stokey, Prescott or any other economist feed into a digital computer for computational purposes if not a computable sequence of rational numbers?

More significantly, from a constructive mathematical point of view the appeal to the Bolzano-Weierstrass theorem in proving the completeness property is the real source of difficulty (see the proof of the Cauchy Completeness Theorem from the Bolzano-Weierstrass property in [55], p.50 and pp.86-7).

Both of these numerical and computational infelicities are 'easily' remedied - in the spirit of the positive title of this paper: 'computing the incomputable, constructing the nonconstructive...'. The computable infelicity is directly and

\footnotetext{
${ }^{34}$ I follow the definitions in [55].

${ }^{35}$ In Körner's exquisitely clear Companion to Analysis, the proof of the demonstration of the completeness of a metric space invokes this property in the form ([46], p. 254; italics added):

'Suppose $\mathbf{a}, \mathbf{b} \in l^{1}$ [where: $l^{1}$ :set of real sequences $\mathbf{a}$, with $\sum_{j=1}^{\infty}\left|a_{j}\right|$ convergent]. We have $\sum_{j=1}^{N}\left|a_{j}+b_{j}\right| \leq \sum_{j=1}^{N}\left|a_{j}\right|+\sum_{j=1}^{N}\left|b_{j}\right| \leq \sum_{j=1}^{\infty}\left|a_{j}\right|+\sum_{j=1}^{\infty}\left|b_{j}\right| ;$ so, since an increasing sequence bounded above tends to a limit, it follows that $\sum_{j=1}^{\infty}\left|a_{j}+b_{j}\right|$ converges and $\mathbf{a}+\mathbf{b} \in l^{1}$.
} 
specifically remedied to give an Effective Contraction Theorem in [39]; the constructivisation of a metric space is achieved with characteristic finesse in [4], pp. 83-86.

In earlier writings I have shown the exact non-constructive content of the standard general equilibrium model; and, a fortiori, in the computable general equilibrium model. I shall, for convenience, paraphrase and repeat some of these arguments here, because the non-constructive infelicities discovered here apply, pari passu, to the application of the Brouwer fix point theorem in [50].

Most mathematical economics textbook ${ }^{36}$ proofs of the Brouwer fix point theorem proceed via some variant of Sperner's Lemma as their underpinning; this lemma, however roundabout, invokes the Bolzano-Weierstrass Theorem ${ }^{37}$. This theorem, as I have mentioned several times above, invokes an undecidable disjunction which is cancelled by an implicit appeal to the axiom of choice. The wording used to camouflage the actual undecidability and submerge reliance on the (full) axiom of choice gives a deceptive appearance of an implementable process for the proof of the Lemma and, hence, the theorem. For example, Starr's clear and detailed presentation of the proof of Brouwer's fix point theorem is based on the excellent and almost elementary exposition in [96] (particularly, pp.424-7). There, in turn, the appeal to the Bolzano-Weierstrass theorem is made almost as with a magician's wand ${ }^{38}$ :

"Making [the] assumption [that given any simplex $S$, there are subdivisions that are arbitrarily fine] we can now finish the proof of Brouwer's fixed-point theorem. We take an infinite sequence of subdivisions of $S$ with mesh, that is, length of the longest onedimensional edge, approaching 0 . From each subdivision, we choose one simplex that carries all labels, and in this simplex we choose a single point. We thus have an infinite sequence of points in the orig-

\footnotetext{
${ }^{36}$ The very recent textbook by Efe Ok on Real Analysis with Economic Applications ([64], Chapter D, $\S 8$, pp. 272-80) proves the Brouwer fix point theorem via the Borsuk Lemma. The proofs of both the Lemma and the theorem are replete with explicit and implicit nonconstructivities, both logical and analytic. See also, below, my comments on retractions.

${ }^{37}$ Just for ease of reading the discussion in this section I state, here, the simplest possible statement of this theorem:
}

Bolzano-Weierstrass Theorem: Every bounded sequence contains a convergent subsequence

${ }^{38}$ In the clear and elementary proof of the Brouwer fix point theorem given in Starr's textbook (op.cit), the appeal to the Bolzano-Weierstrass theorem is made when proving the KKM theorem (p. 62). In Scarf's own elegant text ([82]) invoking of this theorem occurs, during the proof of Brouwer's theorem, on p. 51:

"As the vectors are increasingly refined, a convergent subsequence of subsimplices may be found, which tend in the limit to a single vector $x^{*}$." (italics added)

Scarf is careful to claim that the required subsequence ' $m a y$ be found', but does not claim that it can be found algorithmically. One may wonder: if not found algorithmically, then how? 
inal simplex $S$, and we can choose a subsequence that converges to a single point. This point .. is the limit point of the sequence of all vertices of all the simplexes from which the points of the convergent subsequence were originally chosen." ([96], p.427; all italics, except the first one, are added)

The deceptive use of the word 'choose' in the above description of a processes conveys the impression that the 'choices', in each case, are algorithmically implementable. However, it is only the first use of the word 'choose' and the implied choice - i.e., choosing simplexes from increasingly fine subdivisions that can be algorithmized constructively. The part that invokes the BolzanoWeierstrass theorem, i.e., 'Choosing a subsequence that converges to a single point' - incidentally, this point is the sought after fixed-point of the Brouwer theorem - entails undecidable disjunctions and as long as any proof relies on this aspect of the theorem, it will remain unconstructifiable ${ }^{39}$. Obviously, this is where the (full) axiom of choice is invoked, but never acknowledged.

An alternative textbook approach to demonstrating the Brouwer fix point theorem is by way of the non-retraction theorem (cf. [83], $\S 4)^{40}$. To the best of my knowledge, every demonstration of the Brouwer fix point theorem via a non-retraction theorem proceeds by way of proof by contradiction and an appeal to the law of double negation in infinitary instances ${ }^{41}$. Moreover, unless the retractions are on highly structured spaces, there is no hope whatsoever of devising algorithms to locate the fix points that are non-constructively shown to exist. Since Brouwer's original proof was along these lines, just before he saw the 'light', so to speak, and began repudiating his theorem for its nonintuitionistic and non-constructive nature, it may be useful to make it known to this audience.

Brouwer's proof of his celebrated fix point theorem was indirect in two ways: he proved, first, the following:

Theorem 8 Given a continuous map of the disk onto itself with no fixed points,

${ }^{39}$ Over fifty years ago, when Brouwer returned to the topic of his famous theorem with an Intuitionist version of it, he made the trenchant observation that seems to have escaped the attention of mathematical economists:

"[T]he validity of the Bolzano-Weierstrass theorem [in intuitionism] would make the classical and the intuitionist form of fixed-point theorems equivalent." ([13], p.1).

The invalidity of the Bolzano-Weierstrass theorem in any form of constructivism is due to its reliance on the law of the excluded middle in an infinitary context of choices (cf. also [23], pp. 10-12) (and the implicit invoking of the full force of the axiom of choice).

${ }^{40}$ Scarf's discussion of the version of this theorem is based on results in [33]. However, Hirsch's proof of the Brouwer fix point theorem in [33] is incorrect (cf. [38]) and, therefore, I shall not discuss the non-constructive aspects in the connection between Scarf's combinatorial lemma on labelling a restricted simplex (op.cit, Lemma 3.43) and the non-retraction theorem.

${ }^{41}$ This is also true of the otherwise lucid paper by Kannai on An Elementary Proof of the No-Retraction Theorem ([40]). Kannai makes the important observation (ibid, p. 264) that the no-retraction theorem is, itself, the higher-dimensional analogue of the IVT. That alone should alert the reader of these pages to the nature of the modifications necessary to constructivise it! 
$\exists$ a continuous retraction of the disk to its boundary.

Having proved this, he then took its contrapositive:

Theorem 9 If there is no continuous retraction of the disk to its boundary then there is no continuous map of the disk to itself without a fixed point.

Using the logical principle of equivalence between a proposition and its contrapositive and the law of double negation ( $\nexists$ a continuous map with no fixed point $=\exists$ a continuous map with a fixed point) Brouwer demonstrated the existence of a fixed point for a continuous map of the disk to itself. This latter principle is what makes the proof of the Brouwer fix point theorem via retractions (or the non-retraction theorem) essentially unconstructifiable. Scarf's attempt to discuss the 'relationship between these two theorems [i.e., between the non-retraction and Brouwer fix point theorems] and to interpret [his] combinatorial lemma [on effectively labelling a restricted simplex] as an example of the non-retraction theorem is incongruous. This is because Scarf, too, like the Brouwer at the time of the original proof of his fix-point theorem, uses the full paraphernalia of non-constructive logical principles to link the Brouwer and non-retraction theorems and his combinatorial lemma ${ }^{42}$.

Finally, returning to the determination of $f^{*}(x)$ as the fix point of the operator $T$, in equations (1) and (2) above, but now in the case of mappings between infinite dimensional spaces, Lucas and Stokey 'extend' the Brouwer fix point theorem to the Schauder fix point theorem by adding to the hypotheses of the former the assumption of equicontinuity ${ }^{43}$. This is the additional 'villain' of the piece - compounding the nonconstructivities of undecidable disjunctions due to the Bolzano-Weierstrass theorem and doubly depending on the axiom of choice for it to be useful in proving existence of fix points and routinely in proving the existence of solutions to the IVP problem for ODEs.

The unwary graduate student of economics, with mathematical aptitudes, is introduced to fix points and their application to canonical problems in general equilibrium theory and macroeconomics, via [50], [64], or any number of other equally 'rigorous' books. They in turn, in their innocence, are taught the obvious - and, indeed, the immediate - usefulness of these theorems; they are also taught, expertly, that one moves in a sequence of increased generalizations, whereby one moves from functions that are 'contractions' to those that are not necessarily 'contractions', and then from spaces that are finite to those that are transfinite. All this seems almost exhilaratingly exciting; but the price one pays for all these apparent generalizations - from the alleged Walrasian

\footnotetext{
${ }^{42}$ Scarf uses, in addition, proof by contradiction where, implicitly, LEM (tertium non datur) is also invoked in the context of an infinitary instance (cf. [83], pp. 1026-7).

${ }^{43}$ First introduced, as pointed out above, by Giulio Ascoli in 1884, [3], in the context of problems in the calculus of variations, but later used as the building block for the famous Ascoli-Arzela theorem (although usually referred to in reverse order), ([55], Chapter 5). The Arzela-Ascoli theorem is to the Heine-Borel-Theorem, as the Schauder fix point theorem is to the Brouwer fix point theorem, from the point of view of 'generalisation' of the spaces over which the theorems are valid.
} 
vice of 'counting equations and variables' to prove existence of equilibria - is in terms of numerical meaninglessness and computational impossibilities. It is not as if this is the only way to formalize orthodox economic theory. Even more unfortunate is the fact that we abuse the natural and intuitive feel for numerical meaning and computational content that a modern generation of students, honed in these concepts due to over-familiarity and over-exposure to the digital world, come with, for their education in economics. Contractions and fix points can be devised for domains that preserve numerical meaning and computational content. It is a mystery that this is not taught routinely to students of economics, a subject that has sanctified the notion of equilibrium and its computation.

In passing it may be apposite to point out that Sraffa's much discussed ${ }^{44}$ book, [91], is systematically and almost entirely, and uncompromisingly, constructive. Generations of ill-educated mathematical economists have been 'rigorously proving' propositions in [91], only because they have not had the wisdom or education to have read the book from the point of view of constructive mathematics (see [101]).

\subsubsection{Hahn-Banach Theorem}

"Just as Molière's hero [M.Jourdain] was astonished to discover that he had been speaking prose all his life, some practical men ${ }^{45}$ may not yet have recognized that they work in $Z F+D C$. (To forestall quibbling on this point we could adopt this as our definition of 'practical man').

.....

Of those theorems, such as the Hahn-Banach Theorem ..., whose usual proofs depend on the Axiom of Choice, most can be derived in $Z F+D C$, provided some mild separability conditions are imposed."

Maitland Wright, [51], p. 284; italics added.

Let me conclude this section by showing, in a very general way, the role played by the Hahn-Banach Theorem in proving the crucial 'Second Welfare Theorem' in economics. I shall refer to the way it is presented, proved and discussed in [50] (although I could equally well have chosen the slightly simpler and clearer exposition in [92]). The Second Welfare Theorem establishes the proposition that any Pareto optimum can, for suitably chosen prices, be supported as a competitive equilibrium. The role of the Hahn-Banach theorem in this proposition is in establishing the suitable price system.

\footnotetext{
${ }^{44}$ Also much-maligned by so-called mathematical economists for alleged 're-inventing' of the proverbial 'wheel', claiming that von Neumann in his own celebrated growth model had achieved whatever it was Sraffa achieved in his book, but with impeccable rigour. These claims echo von Neumann's own dismissal of the $\delta$-function as being non-rigorous.

${ }^{45} \mathrm{I}$ am not sure at all that these are the 'practical men' Keynes had in mind in his celebrated concluding paragraph of the General Theory, ([42], p.283)!
} 
Lucas and Stokey state 'their' version of the Hahn-Banach Theorem in the following way ${ }^{46}$ :

Theorem 10 Geometric form of the Hahn-Banch Theorem.

Let $S$ be a normed vector space; let $A, B \subset S$ be convex sets. Assume:

(a). Either $B$ has an interior point and $A \cap \stackrel{\circ}{B}=\emptyset,(\stackrel{\circ}{B}$ : closure of $B)$;

(b). Or, $S$ is finite dimensional and $A \cap B=\emptyset$;

Then: $\exists$ a continuous linear functional $\phi$, not identically zero on $S$, and a constant $c$ s.t:

$\phi(y) \leq c \leq \phi(x), \forall x \in A$ and $\forall y \in B$.

Next, I state the economic part of the problem in merciless telegraphic form as follows:

There are $I$ consumers, indexed $i=1, \ldots, I$;

$S$ is a vector space with the usual norm;

Consumer $i$ chooses from commodity set $X_{i} \subseteq S$, evaluated according to the utility function $u_{i}: X_{i} \rightarrow \Re$;

There are $j$ firms, indexed $j=1, \ldots ., J$;

Choice by firm $j$ is from the technology possibility set, $Y_{j} \subseteq S$; (evaluated along profit maximising lines);

The mathematical structure is represented by the following absolutely standard assumptions:

1. $\forall i, X_{i}$ is convex;

2. $\forall i$, if $x, x^{\prime} \in C_{i}, u_{i}(x)>u_{i}\left(x^{\prime}\right)$, and if $\theta \in(0,1)$, then $u_{i}\left[\theta x+(1-\theta) x^{\prime}\right]>$ $u_{i}\left(x^{\prime}\right)$

3. $\forall i, u_{i}: X_{i} \rightarrow \Re$ is continuous;

4. The set $Y=\sum_{j} Y_{j}$ is convex;

5. Either the set $Y=\sum_{j} Y_{j}$ has an interior point, or $S$ is finite dimensional;

Then, the Second Fundamental Theorem of Welfare Economics is:

Theorem 11 Let assumptions $1-5$ be satisfied; let $\left[\left(x_{i}^{0}\right),\left(y_{j}^{0}\right)\right]$ be a Pareto Optimal allocation; assume, for some $h \in\{\overline{1}, \ldots . . \bar{I}\}, \exists \hat{x}_{h} \in X_{h}$ with $u_{h}\left(\hat{x}_{h}\right)>$ $u_{h}\left(x_{h}^{0}\right)$. Then $\exists$ a continuous linear functional $\phi: S \rightarrow \Re$, not identically zero on $S$, s.t:

(a). $\forall i, x \in X_{i}$ and $u_{i}(x) \geq u_{i}\left(x^{0}\right) \Longrightarrow \phi(x) \geq \phi\left(x_{i}^{0}\right)$;

(b). $\forall j, y \in Y_{j} \Longrightarrow \phi(j) \leq \phi\left(y_{i}^{0}\right)$;

\footnotetext{
${ }^{46}$ Essentially, the 'classical' mathematician's Hahn-Banach theorem guarantees the extension of a bounded linear functional, say $\rho$, from a linear subset $Y$ of a separable normed linear space, $X$, to a functional, $\eta$, on the whole space $X$, with exact preservation of norm; i.e., $|\rho|=|\eta|$. The constructive Hahn-Banach theorem, on the other hand, cannot deliver this pseudo-exactness and preserves the extension as: $|\rho| \leq|\eta|+\varepsilon, \forall \varepsilon>0$. The role of the positive $\varepsilon$ in the constructive version of the Hahn-Banach theorem is elegantly discussed by Nerode, Metakides and Constable in their beautiful piece in the Bishop Memorial Volume ([63], pp. 85-91). Again, compare the difference between the 'classical' IVT and the constructive IVT to get a feel for the role of $\varepsilon$.
} 
Anyone can see, as anyone would have seen and has seen for the last 70 years, that an economic problem has been 'mangled' into a mathematical form to conform to the structure and form of a mathematical theorem. This was the case with the way Nash formulated his problems; the way the Arrow-Debreu formulation of the general equilibrium problem was made famous; and legions of others.

It is a pure mechanical procedure to verify that the assumptions of the economic problem satisfy the conditions of the Hahn-Banach Theorem and, therefore, the powerful Second Fundamental Theorem of Welfare Economics is proved $^{47}$.

It also brings with it the conundrums of the axiom of choice and all the rest of the non-constructivites discussed in the previous section.

Let me end with a simple question: why should an economist force the economic domain to be a normed vector space? Why not a separable normed vector space? Isn't this because of pure ignorance of constructive mathematics and a carelessness about the nature and scope of fundamental economic entities and the domain over which they should be defined?

\subsection{Taming Uncomputabilities}

"The best known model of mechanical computation is (still) the first, introduced by Turing ([99]), and after half a century of study, few doubt the truth of the fundamental Church-Turing Thesis: A function $f: \mathbb{N} \rightarrow \mathbb{N}$ on the natural numbers (or, more generally, on strings from a finite alphabet) is computable in principle exactly when it can be computed by a Turing Machine."

Yiannis N. Moschovakis: What Is an Algorithm? ([60], p.920; italics in the original))

Over the years I have discussed and demonstrated uncomputability in various domains of economics, both micro (rational choice theory) and macro (growth and rational expectations equilibria in overlapping generations models). In general I have used Rice's Theorem, the Unsolvability of the Halting Problem for Turing Machines and the diagonal argument ${ }^{48}$ as the canonical recursion theoretic tools to prove uncomputability propositions in rational choice theory, growth theory, etc.

In this section I want to take a non-standard economic example to discuss uncomputabilities. In recent years my friend and collaborator, Stefano Zambelli, has felicitously and successfully begun to investigate issues in endogenous growth theory using the Busy Beaver as a paradigmatic metaphor ([107], [108]). The discussion in this section is confined to some abstract remarks on the structure,

\footnotetext{
${ }^{47}$ To the best of my knowledge an equivalence between the two, analogous to that between the Brouwer fix point theorem and the Walrasian equilibrium existence theorem, proved by Uzawa ([100]), has not been shown.

${ }^{48} \mathrm{I}$ began using the diagonal argument more confidently in recent years only because I learned its constructive nature rather late in my life (cf., Theorem 4, [4], p. 87).
} 
behaviour and implications of these fascinating repositories of uncomputabilities. Apart from the inspiration from Stefano Zambelli's work in endogenous growth theory, this discussion of the apparently untamable behaviour of Busy Beavers is also a continuation of the discussion of alternative mathematical structures that may be more relevant for the formalization of economic theory than the rather worn model of 'classical' real analysis. In a sense the way I want to use the Busy Beaver is entirely inspired by the idea of non-predictability of entirely determined structures. This way the ad hockery of stochastic assumptions can be reduced or even eliminated in economics. I refer the interested reader to Zambelli's relevant papers to see how the discussion here can be given economic content and relevance.

Tibor Rado introduced and defined a Busy Beaver in his classic paper: "On Non-Computable Numbers" ([73]). Till about the time Rado's paper was published, the standard way of demonstrating the existence of non-computable functions was to diagonalize out of the collection of enumerable computable functions. Rado explicitly stated the basic principle he used to construct a non-computable function:

"The examples of non-computable functions to be discussed below will be well defined in an extremely primitive sense; we shall use only the principle that a non-empty finite set of non-negative integers has a largest element. Furthermore, we shall use this principle only for exceptionally well-defined sets;..."

ibid, p.877; italics added.

What is the connection between this 'extremely primitive case', based on a simple and intuitively acceptable principle and the activities of Busy Beavers, usually defined in the literature ([8]) It is the following. Suppose an arbitrary Turing Machine $(T M)$ is started, scanning a square on a blank, infinite, tape, and it halts after writing $p$ (not necessarily consecutive) $1 \mathrm{~s}$, then the productivity of the TM is $p$. However, any TM, initialised similarly, that does not halt even if it does 'write' some 1s as it continues to operate - is defined to be of productivity 0 . Thus we can define the following total number-theoretic function, $\sum(n)$ for the class of $n$-state $T M s$, denoted by $T M_{n}$ :

Definition $12 \sum(n) \equiv$ Maximum productivity of any member of $T M_{n}$ and is called the Busy Beaver Function.

Definition 13 Members of the Busy Beaver Club are those n-card TMs (for any given n), when initialised on a blank, two-way infinite tape, that write the maximum number of $1 s$ (not necessarily consecutive) on an otherwise blank tape and halt. The Busy Beaver function is $\sum(n)$ and the shift function associated with this is given by $S(n)$.

We know, from an easily derivable formula, that the number of n-card TMs, $\forall n$, is a finite integer value. Hence, $\sum(n)$ is, obviously, a total number-theoretic function. It follows, therefore, that $\sum(n)$ is the largest number in a finite set 
of natural numbers and the finite set of natural numbers, in turn, is the set of productivities of n-card TMs. This, then, is how Rado uses the principle that a non-empty finite set of non-negative integers has a largest element.

How, from this intuitive principle, does one go on to construct a noncomputable function without using a diagonal argument. For Rado explicitly states that he eschews any reliance on this old workhorse ${ }^{49}$ :

"It may be of interest to note that we shall not use an enumeration of computable functions to show that our examples are non-computable functions. Thus, in this sense, we do not use the diagonal process."

$$
\text { ibid, p.877; italics added. }
$$

In fact the next step taken by Rado, after defining productivity and $\sum(n)$, is to invoke, implicitly, the implications of the Berry Paradox, in the special context of computable functions as $\mathrm{TMs}^{50}$. The Berry Paradox deals with the notion of finite definability and is semantical in nature. Therefore, it is possible to describe the construction of non-computable functions using a simple programming language. Now, consider the following:

Proposition $14 \sum(n)$, and a fortiori $S(n)$, are uncomputable.

I shall give a slightly unconventional proof of this well-known proposition in such a way that makes it possible to exploit analogies between $\sum(n)$ and Chaitin's $\Omega$. The reasons for doing this will become evident in the sequel. It was noted that:

"... [T] here is the basic fact of the noncomputability of $\sum(n)$, which implies that no single finite computer program exists that will furnish the value of $\sum(n)$ for every $n$.

In the absence (at present) of a formal concept of 'noncalculability' for individual well-defined integers, it is of course not possible to state in precise form the conjecture that there exist values of $n$ for which $\sum(n)$ is not effectively calculable."

[48]Lin and Rado (1965), p.212; italics added.

Is there a formal concept of randomness 'for individual well-defined integers'? There is and it is provided by Kolmogorov's Algorithmic Complexity

${ }^{49}$ However, an eminent scholar of Busy Beaver problems asserts the contrary

"Using essentially a diagonal argument he[Rado] demonstrated that if $f$ is some computable function then there exists a positive integer $n$ such that $\sum(k)>$ $f(k)$ for all $k>n . "([9], p .239 ;$ italics added $)$ :

Since Rado invokes, albeit implicitly, the Berry Paradox, Brady's remark cannot be quite correct, except by some considerable stretch of the imagination.

${ }^{50}$ Rado, via the Berry Paradox to non-computable functions; Gödel via the Liar and Richard Paradoxes to incompleteness; Chaitin via the Berry Paradox to algorithmic complexity; Turing via Cantor's Procedure to uncomputability; such are the ways in which great mathematical sagas have been grounded in paradoxes. 
Theory and Chaitin's Algorithmic Information Theory. If it is possible to define the randomness of an integer why is it not possible to define, formally, the "concept of 'noncalculability' for individual well-defined integers"? My basic conjecture is that the 'noncalculability' of Rado's $\sum(n)$ is exactly analogous to the randomness of Chaitin's $\Omega$ (cf [14], Part II, pp.80-82). Chaitin constructed his uncomputable and random $\Omega$, exploiting a form of the Berry Paradox; I shall, however, exploit it for deriving the crucial contradiction in proving the uncomputability of $\sum(n)$. The reason for emphasising this link between Chaitin's $\Omega$ and Rado's $\sum(n)$ via the Berry Paradox is, partly, to make a point of the status of proofs by contradiction when impredicative definitions are involved.

A few unnecessary cobwebs must, first, be cleared away. There are those who claim - even eminent 'Busy Beaver scholars' - that a variant of the diagonal method was used to 'construct' $\sum(n)$, despite an explicit statement to the contrary by Rado himself (see above and op.cit, p.877). Then, there are equally eminent textbook writers who assert that Rado's construction of the Busy Beaver was based on Richard's paradox ${ }^{51}$. Neither of these assertions are quite correct. Rado's construction uses, albeit implicitly, the Berry paradox, which being semantical is not directly related to the more obviously 'diagonal' based Cantor and Russell logical paradoxes.

So far as I know, proof by contradiction has been the only way that the uncomputability of $\sum(n)$ has been 'rigorously' demonstrated. Even in proofs where there seems to be an explicit 'construction', for example in the well-known and clear demonstration in Boolos and Jeffrey (op.cit, especially chapter 4), it is, in reality, by way of a 'thought experiment' to justify the assumption of the contrary to the hypothesis in the theorem. I am never sure that such proofs convey the real meaning of a theorem, particularly when the theorem asserts that something cannot be constructed, decided or computed. Therefore, before I myself give a 'proof by contradiction' of the uncomputability of $\sum(n)$, it may be useful to discuss the intuitive content of the meaning of this strange ${ }^{52}$ result.

The intuitive reasons normally given to explain the uncomputability of $\sum(n)$ is that it 'grows' too fast to be computed by the standard operations on intuitively acceptable and formally computable elementary functions. A brilliant

\footnotetext{
${ }^{51}$ Thus, Neil Jones (1997), in an otherwise exceptionally well-written and admirably pedagogical textbook on 'Computability and Complexity' states (pp.16-7; second set of italics added):

"The busy beaver function ..., due to Rado and related to the Richard paradox, is mathematically well-defined, but based on certain reasonable assumptions about the language used to express computation"

Two minor caveats may be called for to make this assertion more acceptable. Firstly, it is not $\sum(n)$ that is 'related to the Richard paradox but the method of proof; secondly, in a convoluted way, it is not incorrect to state what Jones has asserted simply because the Richard paradox is related to the Berry paradox, since they are both semantic in origin (using Ramsey's distinction between logical and semantic paradoxes; cf. Ramsey, 1926 and Kleene, 1967, esp. pp. 188-190).

52 'Strange' only because, as noted by Rado himself, $\sum(n)$ is an 'exceptionally well-defined number', in that its existence follows from a simple well-ordering principle: that a finite set of numbers has a maximum.
} 
and graphic description of this idea is given by Dewdney in his fine expository piece on Busy Beavers:

"The function $\sum(n)$ has an extraordinary property: it is not computable. It simply grows too fast. From the first four values of $\sum(n)$ - namely $1,4,6$ and 13 - it might seem that the rate of growth is only moderate. .... On the other hand, [there is] a 12state machine that generates so many 1's that the number must be expressed by the following mind-boggling formula:

$$
6 \times 4096^{4096^{4096} \cdot{ }^{4096^{4}}}
$$

The number 4,096 appears 166 times in the formula, 162 times in the 'twilight zone' represented by the three dots. The formula can be evaluated from the top down: first raise 4,096 to the fourth power, then raise 4,096 to the power of the resulting number, then raise 4,096 to the power of that number, and so on. When you reach the bottom, multiply by 6 .

Anyone whose mind does not boggle when confronted by a string of 1 s that long is welcome to construct an even bigger number. Write down any formula you like in which numbers are multiplied or raised to a power; you may even replace any of the numbers with $n$. No matter what formula you devise, for some value of $n$ that is large enough the n-state busy beaver will produce more 1's than the formula specifies. It follows that $\sum(n)$ cannot be calculated for arbitrary large values of $n$. The best one can do is to calculate $\sum(n)$ for some small, fixed value of $n$."

Dewdney (op.cit), pp. 10-11; italics added.

But there seems another catch: "No matter what formula [we] devise, for some value of $n$ that is large enough the n-state busy beaver will produce more 1 's than the formula specifies"! Now, in our 'world' of discourse, all formulas are computation rules, programme specifications. Does this mean, whatever computation rule we may devise, whatever programming language we use, is not powerful enough conceptually, computationally, syntactically or semantically to tame $\sum(n)$ ? How, we may then ask, was randomness tamed by algorithmic complexity theory and algorithmic information theory? Is there a lesson to be learned and applied from that experience?

There is, however, an almost exact analogy, in the history of classical recursion theory, to the role played by Rado's $\sum(n)$ vis-à-vis TMs, programming semantics and, via the Church-Turing thesis, the partial recursive functions. It is the place of the Ackerman function vis-à-vis primitive recursion. The analogies are uncannily similar and I shall pursue it to elucidate the difficulties one faces with any attempt to tame $\sum(n)$.

By an enumeration of the primitive recursive functions, coupled to a simple diagonalization procedure, the 'existence' of a computable function that was 
total but not primitive recursive was easily shown. This was similar to the situation with the demonstration of the 'existence' of uncomputable functions via diagonalization out of an enumeration of the computable function. Rado, as I pointed out above, expressed dissatisfaction at this 'nonconstructive' existence demonstration and endeavoured to 'construct', explicitly, an intuitively acceptable function that was, nevertheless, shown to be uncomputable. Similarly, the Ackerman function was accepted as an intuitively computable function that was not primitive recursive. This led to an enlargement of the class of intuitively acceptable computable function and a new operation, minimalization, was introduced to the old repertoire of composition and primitive recursion and the partial recursive functions were defined. Is the lesson from that important episode in the history of classical recursion theory that we can try to enlarge the rules of operation or expand the class of initial functions so as to bring into the fold of the computable also $\sum(n)$ ? The problem, however, with $\sum(n)$ vis-à-vis TMs, partial recursive functions, etc., is that no one seems to have been able to figure out a 'new' operation that can tame the activities of the Busy Beaver.

Let me now prove Proposition 14. I shall therefore attempt to present an alternative perspective for which it is necessary to restate Proposition 1 in a slightly different, but formally equivalent, way. Before I present this alternative version of Proposition 1, consider the following standard result in classical recursion theory ([59], p.156, theorem 5.5):

Theorem 15 The length of the shortest program that prints $n$ and halts is not computable.

Compare the form and content of the above theorem with the following ([15], p.4):

Theorem 16 The first positive integer that can be proved (relative to a given formal axiomatic system) to have the property that it cannot be specified (relative to a given Universal Turing Machine) by a computer program with less than $N$ bits is uncomputable.

Next, compare both of the above theorems with the following version of Berry's Paradox (Russell, 1908, p.222). Consider the least integer not nameable in less than nineteen syllables. This refers to the particular number 111,777. But the italicised expression also names an integer and contains eighteen syllables! In other words, the 'least integer not nameable in less than nineteen syllables', i.e., 111,777 , can be named in eighteen syllables. This is the kind of contradiction that is exploited in the proof of the above two theorems. For example, in Theorem 16, the strategy of the proof is to show that there is, in fact, a computer program of length $\log _{2} N+$ constant $=N$, for sufficiently large $N$, which will specify the same number and hence to display a contradiction. Exactly the same strategy is employed in proving Theorem, 15.

Now here is my alternative statement of Proposition 1:

Proposition $\mathbf{1 7}$ For any given n, the largest value that can be printed by a program of length (at most) $n$, is uncomputable. 
Remark 18 Clearly, Proposition 17 is a kind of converse of Theorem 15. Hence, a proof of the former will only need to mimic the strategy adopted to prove the latter. The affinity with the form of the Berry Paradox is also evident. And, with respect to Theorem 16, if we replace the phrase 'first positive integer' with the 'largest positive integer', then the analogy with it is also clear.

Proof. Suppose $\sum(n)$ is computable. Define an auxiliary constant-valued

function $f$ by minimalization such that, for any given $m$ :

$$
f(m)=\mu j \text { and } \sum(j) \geq m
$$

The minimalized function $f$ returns the natural number $j$ such that no program of length less than $m$ prints $j$ and halts. Clearly, the length of the program for $f \leq$ constants + the bits necessary to code $m$ so that $f$ can make sure that $\sum(j) \geq m$. The latter value is at $\operatorname{most} \log _{2} m$. Denote the constant value as $c$. Then the length of the program to implement $f$ is less that $\left|c+\log _{2} m\right|$. If, now, $m$ is chosen large enough to make sure that it is sufficiently greater than $\log _{2} m$ then for such a choice, say $m_{0}, f$ computes the least value $j$ such that no program of length less than $m_{0}$ can print $j$. But $m_{0}$ was chosen to guarantee that the program for computing $f$ was, in fact, less than $m_{0}$. This contradiction implies that $\sum(n)$ is computable.

Proving, by contradiction, the uncomputability of $\sum(n)$, exploits the full force and potentialities of the Berry Paradox. Where lies the paradoxical aspect of the Berry Paradox? It lies in what was called impredicative definitions by Poincaré, definitions that involve some aspect of self-reference where, however, the paradox arises from allowing totalities to be members of themselves. Russell, inspired partly by Poincaré, was led to develop his theory of types to tackle these paradoxes. This is not the place to go into the full and fascinating details of these issues but it may be pertinent to repeat some of Russell's pertinent observations on at least the Berry Paradox.

Why are we able to resort to proof by contradiction to demonstrate the validity of any proposition that is related to the Berry Paradox? It is, as Russell pointed out almost a century ago, because (op.cit., p.223; italics in original) :

"In the cases of names and definitions [i.e., the Berry and Richard Paradoxes], the paradoxes result from considering nonnameability and indefinability as elements of names and definitions. .... In each contradiction something is said about all cases of some kind, and from what is said a new case seems to be generated, which both is and is not of the same kind as the cases of which all were concerned in what was said."

More particularly, regarding the Berry (and Richard) Paradox: 
"The least integer not nameable in fewer than nineteen syllables' involves the totality of names, for it is 'the least integer such that all names either do not apply to it or have more than nineteen syllables'. Hence we assume, in obtaining the contradiction, that a phrase containing 'all names' is itself a name, though it appears from the contradiction that it cannot be one of the names which were supposed to be all the names there are. Hence, 'all names' is an illegitimate notion."

ibid, p.224; italics added.

I do not know of any proof of the uncomputability of $\sum(n)$ that does not invoke some version of this 'illegitimate notion'. On the other hand, Chaitin, in constructing $\Omega$, did circumvent the illegitimacy by restricting the scope of 'nameability' or 'definability'. He achieved this restriction by specifying 'nameability' and definability' to satisfy the requirement of being calculated outputs of a suitably defined UTM.

What are to make of this situation? Perhaps there is no need to 'prove' that $\sum(n)$ is uncomputable; it may be sufficient to demonstrate that dangerous curses of dimensionality may lie wrapped in the enigma that is the Busy Beaver. It may well be that we should resort to a Linnean philosophy of investigation: careful study of small, selected, well structured examples and diligent classification. This is not unlike the practice of the dynamical system theorist who wishes to tame the vagaries of nonlinear systems.

I shall, however, suggest another alternative in the face of this humbling of formalism and its methods, inflicted by the Busy Beaver. This alternative suggestion, entirely based on an extremely interesting 'attack' on the vagaries of $\sum(n)$ by Greenleaf ([32]), when supplemented with Chaitin's careful circumvention of the Berry Paradox, results in a partial humbling of the Busy Beaver!

The strategy carried out by Russell and others (Poincaré, Weyl etc.) - to banish impredicative definitions from mathematical discourse - may smack of a mildly defeatist attitude. Paradoxes play the kind of role counter-examples play in sharpening an understanding of the scope of mathematical theorems. A world of mathematical semantics without paradoxes may well be poorer even from an epistemological viewpoint.

There is a more elegant way out of this dilemma. The idea for this alternative way was motivated by trying to define the value of $\sum(n)$, for any given $n$, in such a way that it will dampen its growth rate. To put it more prosaically, I want to drug the Busy Beaver into slowing down its eagerness! This is my interpretation of the elegant approach suggested by Greenleaf (op.cit), which is based on a philosophy of mathematics that is particularly significant in any attempt to tame the behaviour of $\sum(n)$, without losing the rich insights into emergent computations that such behaviour gives rise to. Let me summarize, first, the mathematical philosophy that underpins Greenleaf's concrete suggestions to manage a meaningful study of $\sum(n)$ :

- The triple \{assumption, proof, conclusion $\}$ can be understood in 
terms of $\{$ input data, algorithm, output data $\}$.

- Mathematics is best regarded as a very high level programming language.

- In constructive, computable ${ }^{53}$ and (constructive) nonstandard analysis, every proof is an algorithm.

- To understand a theorem (in any kind of mathematics) in algorithmic terms, represent the assumptions as input data and the conclusions as output data. Then try to convert the proof into an algorithm which will take in the input and produce the desired output. If you are unable to do this, it is probably because the proof relies essentially on the law of excluded middle.

- If we take algorithms and data structures to be fundamental, then it is natural to define and understand functions in these terms. The phrase "noncomputable function" then becomes problematic, and the understanding which sees almost all functions as non-computable becomes mysterious. If a function does not correspond to an algorithm, what can it be? There is no higher court corresponding to the set theory of logical mathematics.

- We shall take the stand that functions are, by definition, computable, and then test those phenomena which are standardly taken as evidence for the existence of non-computable functions, to see if we need to yield any ground.

- Given a putative function $f$ - say Rado's $\sum(n)$ - we do not ask "Is it computable?" but rather "What are the data types of the domain and of the range?" This question will often have more than one natural answer, and we will need to consider both restricted and expanded domain/range pairs. Distinguishing between these pairs will require that we reject excluded middle for undecidable propositions. If you attempt to pair an expanded domain for $f$ with a restricted range, you will come to the conclusion that $f$ is non-computable.

To use this alternative vision of mathematics and mathematical activity towards a reinterpretation and taming of the activities of the Busy Beaver , it will be helpful to begin by considering the rigorous definition of a real number, either via a Dedekind cut or via a Cauchy sequence of rationals ${ }^{54}$. In the former case the set $\mathbb{R}$ of real numbers is defined as the collection of all Dedekind cuts and the elements of $\mathbb{R}$ are then defined as certain subsets of $\mathbb{Q}$. In the latter case, real numbers are equivalence classes of Cauchy sequences of rational numbers. There is, of course, more algorithmic content in the definition of $\mathbb{R}$

\footnotetext{
${ }^{53}$ In the case of affirmative, existence, proofs. Universal negative propositions use the full paraphernalia of classical logic, including the law of the excluded middle.

${ }^{54}$ I shall not consider the other two methods: via nested intervals or by means of decimal expansion to a base, say $b$, where $b$ is an integer $>0$. Goodstein's uniform calculus, for example, proceeds by way of the latter method.
} 
as equivalence classes of Cauchy sequences of rational numbers, but the point is that in both definitions a real number is characterized in terms of a collection of rational numbers.

A real number can only be determined up to a pre-assigned degree of approximation. Some real numbers are hard to describe, i.e., compute; they are algorithmically highly complex. It takes time to determine them even to low levels of approximation. If, taking a cue from this setup, we define $\sum(n)$ in some equivalent way, we would kill the proverbial two birds with one stone: on the one hand, slow down the growth rate of $\sum(n)$; on the other, make it analogous to Chaitin's $\Omega$. The idea would be to determine the binary code of any given (combinatorial) object and define its Busy Beaver complexity as the minimum $k$-card TM that would print the number of $1 \mathrm{~s}$ in that code (perhaps separated by ordered blanks). In this way, we might be able to free the definition of complexity even from its underpinnings on any concept of information; instead defining it dynamically in a natural setting. In other words, every combinatorial object (i.e., algorithmic object) will be the output of some Busy Beaver. The complexity of the given object is the algorithmic description of the minimum Busy Beaver that would print its code and halt. Obviously, this complexity, too, would be uncomputable.

Now to return to Greenleaf's philosophy of mathematics and its use in taming the activities of the Busy Beaver, we exploit the analogies inherent in another aspect of the real numbers: their definitions as sets of rational numbers plus an approximation scheme and degree (for example in the Cauchy characterization of $\mathbb{R}$ ). The first question in this endeavour, given the above summary of Greenleaf's mathematical philosophy, what is the appropriate data type for studying $\sum(n)$ ? It is in answering this question that the analogy of defining the members of $\mathbb{R}$ as subsets of $\mathbb{Q}$ will be exploited. It may be best to state Greenleaf's 'solution' before even describing its structure:

"The busy beaver function $b b\left[\equiv \sum(n)\right]$ becomes computable when its domain and range are properly defined. When the domain is taken to be $\mathbb{N}$, the range will be the set of 'weak integers', a superset of $\mathbb{N} \ldots$...

ibid, p.226; italics added.

The 'weak integers', in essence, 'weaken' the over-enthusiasm of the Busy Beavers. 'Weak integers' are constructed and defined relative to a given UTM in the following way:

Step 1:

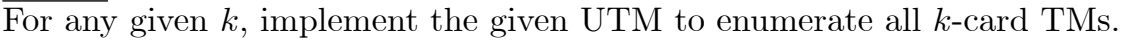

Step 2:

Next, execute each $k$-card TM from the enumerated list on a standard twoway infinite blank tape for some given, arbitrarily long, time interval.

Step 3:

Whenever a Busy Beaver is found, its productivity is listed on an auxiliary tape. 
Step 4:

$\overline{\sum(k)}: \mathbb{N} \rightarrow\{\mathbb{N} \times \mathbb{N} ; U T M\}$

where the range is given by a pair constructed as follows: to each element in the enumerated list of $k$-state TMs, associate the result, an integer, which is possibly the determined productivity associated with it or a 'temporary' value obtained from an Oracle computation, by the given $\mathrm{UTM}^{55}$.

Thus the set of 'weak integers', say $\Xi$, are approximations from below in the same sense in which semi-computable functions are defined to approximate from above and below and the analogy goes even further: naturally, $\Xi$, too, will be a recursively enumerable set that is not recursive. The standard integers will be the singleton sets and, therefore, $\mathbb{N} \subset \Xi$. Natural order relations can be defined over $\Xi$, but I leave the interested reader to consult Greenleaf (op.cit) for such details.

I have tried to suggest a mathematical way of taming uncomputabilities by identifying their nature. Before minimalization was introduced to tame the growth of primitive recursive functions, the explosion of the behaviour of the Akerman function was a perplexity. After the introduction by Rado of the Busy Beaver's industriousness, the perplexity has been renewed. Whether the suggestion above is a way out of, and beyond, this renewed perplexity, I am not sure. But I am convinced of its relevance for the formalization of economic theory and for understanding the paradoxes of endogenous growth, as perceived by Zambelli's modelling of endogenous forces non-stochastically

\section{Reconstructing Mathematical Economics}

"Fred Richman [concludes] .. that once a mathematician sees the distinction between constructive and nonconstructive mathematics, he or she will choose the former. That conclusion, if extrapolated further than Professor Richman intended, suggests that any mathematician can learn constructivism easily if he or she do desires.

But in fact constructivism is unusually difficult to learn. Learning most mathematical subjects merely involves adding to one's knowledge, but learning constructivism involves modifying all aspects of one's knowledge: theorems, methods of reasoning, technical vocabulary, and even the use of everyday words that do not seem technical, such as 'or'."

Eric Schechter ([85], p.51)

Learning constructive mathematics may well require an aesthetic sensibility which will have to be nurtured with deliberation, rather like the way the 'new mathematics' of Bourbakism penetrated education curricula at elementary levels to change visions, expectations and hopes. Few remember the noble motivations of the young Frenchman who survived or who remembered the legions of lost

\footnotetext{
${ }^{55}$ At this last stage of the last step I part company with Greenleaf, mainly because I wish to retain the analogy with Chaitin's $\Omega$.
} 
compatriots in the Great War and vowed to create a sanitised mathematics that will not be manipulated for ulterior purposes. Few will be motivated by the foundational crises and the battles between intellectual giants, fighting for the soul of the foundations of mathematics, that led to the emergence of Constructive Mathematics as we know it today. Lip service is paid to the battles, sometimes undignified, between Hilbert and Brouwer and between Kronecker and Cantor, but now, after Bishop and others, Constructive Mathematics is a serious discipline in its own right.

A legitimate question, one that a classically trained mathematician may ask a Constructive Mathematician, is the following. 'Alright', she might say; 'I'll try to begin my re-education by reading Bishop ([4]); but can you give me some methodological guidelines?' By this the sympathetic 'classical' mathematician may mean the following: 'give me a hint, or a set of precepts, that would allow me to understand the extent to which 'classical' mathematics is 'idealized' constructive mathematics' (strenuously avoiding the seemingly condescending statement that constructive mathematics is the approximating version of 'classical' mathematics, implying that the latter is ideal mathematics). To this Bishop has already given helpful answers in terms of the Principle of Omniscience (PO), Limited Principle of Omniscience (LPO), Lesser Limited Principle of Omniscience (LLPO), their conjoint use with various versions of the axiom of choice, and their connections with the law of the excluded middle $(L E M)$, the law of double negation $(\neg \longrightarrow P \Longrightarrow P)$, etc., and, hence, also modes of proof - whether it is a proof by contradiction, by contraposition, and so on (cf. [4], pp. 8-11; [12], pp. 9-11). Thus, Bishop was able to say, almost categorically ([4], p.9, italics added) :

"The proof that the real numbers can be well ordered is an instance of a proof in which a sweeping use of the principle of omniscience is combined with an appeal to the axiom of choice. Such proofs offer little hope of constructivization."

It may be useful to point out that LPO is a special case of $L E M$ and IVT is equivalent to LLPO; the 'classical' Hahn-Banach theorem $\Longrightarrow L L P O$; and so on. Such an understanding shows what to look for, and where, in a 'classical' proof so as to constructivise it. This was easy in the case of IVT; a little less easy in the case of the Hahn-Banach theorem; much more difficult in the case of the Brouwer fix point theorem, etc.

But, if Schechter is right, and constructive mathematics is 'unusually difficult to learn' by mathematicians (and, perhaps, equally, or even more difficult, to teach), then imagine how much more difficult the task is in economics? In particular, when unlearned and obviously ignorant and prejudiced statements are made by reputable economists, but also by silly mathematical economists. On the one hand, we have two distinguished economists claiming constructivity and computability of models that are clearly neither the one nor the other:

"The major result of postwar mathematical general equilibrium theory has been to demonstrate the existence of ... an equilibrium 
by showing the applicability of mathematical fixed point theorems to economic models. ... Since applying general equilibrium models to policy issues involves computing equilibria, these fixed point theorems are important: It is essential to know that an equilibrium exists for a given model before attempting to compute that equilibrium. .....

$\ldots$

The weakness of such applications is twofold. First, they provide non-constructive rather than constructive proofs of the existence of equilibrium; that is, they show that equilibria exist but do not provide techniques by which equilibria can actually be determined. Second, existence per se has no policy significance. .... Thus, fixed point theorems are only relevant in testing the logical consistency of models prior to the models' use in comparative static policy analysis; such theorems do not provide insights as to how economic behavior will actually change when policies change. They can only be employed in this way if they can be made constructive (i.e., be used to find actual equilibria). The extension of the Brouwer and Kakutani fixed point theorems in this direction is what underlies the work of Scarf .... on fixed point algorithms ...."

[89], p.12 \& pp. 20-1; italics added

Surely, the blame should be placed on the use of a mathematical framework which encourages - and thrives on - this separation between proving existence and determining it? This is exactly the core difference between 'classical' and constructive mathematics. Mathematical Economics is replete with appeals to fix point theorems because economics is obsessed with the logical problem of equilibrium existence. But policy and any other interesting implication of such a mythical equilibrium calls forth constructive processes, hopefully analogous to the actual individual and institutional ones, determining it. The melancholy fact that even the good intentions and claims in the above quote are technically incorrect, as shown above, in this paper.

On the other hand, we have pseudo-sophisticated authors of indigestible texts on 'mathematics for economists' advising students to learn constructive mathematics in their 'spare time', even while the author's own understanding of the subject is hopelessly wrong:

"If you want to learn about intuitionism in mathematics, I suggest reading - in your spare time, please - the four articles by Heyting and Brouwer in Benacerraf and Putnam ..."

[64], p. 279, footnote, 47; italics added.

This book is liberally endowed with inanities regarding the axiom of choice, functions as rules and many other things dealt with above, in this paper - and the book is almost paradigmatic of the way mathematics is taught in advanced economic curricula. The poor economist, trying to learn the mathematics relevant 
to hone a natural economic intuition, is fed absolute rubbish with pretensions of scholarship where none exist.

The mathematical economist seems to have lost touch with the history of the subject, with the impulses that motivated the pioneers when they sought to formalize economic theory. Thus we have a distinguished mathematician, writing together with a reputable economist, claiming that the natural domain of economic theory is that which is determined by real analysis:

"Computing with real numbers offers some important advantages in the context of scientific computing. ... it is also relevant to applications in economic theory. Economic models typically use real variables and functions of them. A model of computing in which the elementary operations are functions of real variables allows the model to be directly applied to standard economic models, without requiring an analysis of approximations in each application."

[61], pp. 1-2; italics added.

We are, of course, not told why 'economic models typically use real variables and functions of them'; nor are we told why 'the important advantages ... of scientific computing offers for computing with real numbers' (as advanced by Blum and others, for example in [7]) has any relevance for economic modelling where the variable are naturally in $\mathbb{N}, \mathbb{Q}$, or $\mathbb{Z}$. Moreover, the book appeals to the results Kolmogorov and Arnold obtained when resolving (negatively) Hilbert's conjecture as expressed in the 13th of his famous problems of 1900 . But the authors do not seem to have understood the difference between exact approximations and precise computations. Had they understood it, they could have advocated economic modelling with Interval Analysis ([58]), Constructive Analysis ([4]) or Computable Analysis ([1]) - or even Nonstandard Analysis ${ }^{56}$. But this requires harder work at a more fundamental economic theoretical level, in addition to learning a whole new mathematics. So, we are condemned to repeat the mistakes of accidental commitments in the past, rather like being trapped in the clutches of $Q W E R T Y$.

I don't think, in fact, it will be as difficult to teach constructive and computable analysis to economists, from ground zero. The young who are computer literate can be taught calculus the way Rosser tried to introduce functions as a rule in the sense in which it would become the foundations for the same notion at the higher level of the $\lambda$-calculus and then on to programming languages. The young who are taught the IVT ab initio will, surely, think it most natural, especially if they are sitting at a terminal while being taught it.

Above all, the lesson I have learned while writing this paper - through the 'brain in the hand' - is a lesson that I did not expect I would try to make a case for when I set out to write it. I now feel the non-constructive mathematical formalism of economic theory is the main culprit in infesting the subject with

\footnotetext{
${ }^{56}$ The embarrassing mistakes in the bibliography in [61] crowns its misleading contents, quite appropriately. The most irritating examples of the bibliographic mistakes were the incorrect titles of two of the classic books on approximation theory - those by Lorentz and Vitushkin.
} 
undecidabilities, uncomputabilities and unsolvabilities. I am less confident than I was before writing this paper that these conundrums are intrinsic to the subject. Institutional mechanisms evolve, naturally, to confront and resolve these difficulties - in spite of the analytical paralysis shown by mathematical economists. I recall a characteristically pungent answer by Ulam to a provocative question put by Gian-Carlo Rota ([80], pp.2-3; italics added):

"What makes you so sure that mathematical logic corresponds to the way we think? You are suffering from what the French call a 'deformation professionelle'. Look at the bridge over there! It was built following logical principles. Suppose that a contradiction was to be found in set theory. Do you honestly believe that the bridge might then fall down?"

We have lost the tradition - perhaps we never had it - of the kind of methodologies fostered and practised by the Feynmans and the Diracs in theoretical physics, whereby the perplexities of the natural sciences and the world it observes and studies, and of which it is a part, are resolved by imaginative mathematics, not shackled by any kind of orthodox strictures of the pure mathematician. We, in mathematical economics, have constrained our visions and our tools and bent our concepts to fit an unfitting mathematics and generated undecidable, uncomputable and non-constructive conundrums that are unnatural for the subject.

Gödel thought - and Feferman disagreed - that new axioms will need to be developed and new axiomatic thinking fostered, to tame unnatural incompleteness. Gödel also thought that an exact metaphysics was possible. I find myself on Ferferman's side - and, therefore, also on Dirac's side - on the question of the need for new axioms - but from the point of view of formal economic theory. But I find myself agreeing with Gödel that, in the face of unnatural perplexities, the human mind will strive to resolve them by developing thought processes and systems of thought that will allow them to be seen for what they are: paradoxes to be used as spurs for new adventures in intellectual journeys.

It is appropriate, at this Conference in Honour the 'greatest logician since Aristotle', that I end my contribution reflecting on the words of Gödel, words that many distinguished living logicians - like Solomon Feferman - think encapsulated his philosophy, and guided his epistemology ([25], p. 103):

"Not only were Gödel's results stunning, but also his own explanation of why they hold was surprising. .. [I]t expressed a fundamental conviction of Gödel's which he reiterated throughout the rest of his life... ."

The explained conviction was expressed with characteristic guardedness and precision ([29], p.191, quotation from the English translation, [30] ; italics added):

"[T]he true reason for the incompleteness inherent in all formal systems of mathematics is that the formation of ever higher types can 
be continued into the transfinite ... [since] the undecidable propositions constructed here become decidable whenever appropriate higher types are added." 


\section{References}

[1] Aberth, Oliver, (2001), Computable Calculus, Academic Press, San diego \& London.

[2] Arrow, Kenneth. J, (1986): Rationality of Self and Others in an Economic System, Journal of Business, Vol. 59, pp. S 385-S 400.

[3] Ascoli, Giulio, 1884, Le curve limite di una varietà data di curve', Accademia nazionale dei Licei, memorie, Classe di Scienze Fisiche, Mathematiche e Naturale, Vol, 3, \#18, pp.521-586.

[4] Bishop, Errett A, (1967), Foundations of Constructive Analysis, McGraw-Hill Book Company, New York.

[5] Bishop, Errett A, (1985), Schizophrenia in Contemporary Mathematics, in: Errett Bishop - Reflections on Him and His Research, pp. 1-32, edited by Murray Rosenblatt, Contemporary Mathematics, Vol. 39, American Mathematical Society, Providence, Rhode Island.

[6] Bishop, Errett \& Douglas Bridges, (1985), Constructive Analysis, Springer-Verlag, Berlin \& Heidelberg.

[7] Blum, Lenore, Felipe Cucker, Michael Shub and Steve Smale (1998), Complexity and Real Computation, Springer Verlag, New York.

[8] Boolos, George. S \& Richard C. Jeffrey (1989): Computability and Logic (Third Edition), Cambridge University Press, Cambridge.

[9] Brady, Allen. H, (1994), The Busy Beaver Game and the Meaning of Life, pp.237-54, in: The Universal Turing Machine - A Half-Century Survey, Second Edition, Springer-Verlag, Heidelberg, 1994.

[10] Bridges, Douglas (1999), Constructive Methods in Mathematical Economics, in: Mathematical Utility Theory - Utility Function Models, and Applications in the Social Sciences edited by gerhard Herden, Norbert Knoche, Christian Seidl and Walter Trockel, Zeitschrift für Nationalökonomie, Supplement 8, pp.1-21.

[11] Bridges, Douglas \& Fred Richman, (1987), Varieties of Constructive Mathematics, Cambridge University Press, cambridge.

[12] Bridges, Douglas \& Luminiţa Vîţă, (2006), Techniques of Constructive Analysis, Springer Science+Business Media, New York.

[13] Brouwer, Luitzen E. J (1952), An Intuitionist Correction of the FixedPoint Theorem on the Sphere, Proceedings of the Royal Society London, Vol. 213 (5 June 1952), pp.1-2. 
[14] Chaitin, Gregory. J (1990), Computing the Busy Beaver Function, in:Information, Randomness \& Incompleteness - Papers on Algorithmic Information Theory (Second Edition), World Scientific, New Jersey.

[15] Chaitin, Gregory. J (1995), The Berry Paradox, Complexity, Vol.1, No.1, pp.26-30.

[16] Church, Alonzo (1956), Introduction to Mathematical Logic, Princeton University Press, Princeton, New Jersey.

[17] Cooley, Thomas. F \& Edward C. Prescott, (1995), Economic Growth and Business Cycles, in: Frontiers of Business Cycle Research edited by Thomas F. Cooley, Chapter 1, pp.1-38, Princeton University Press, Princeton, New Jersey.

[18] da Costa, Newton. C. A \& Francisco Doria (1991), Undecidability and Incompleteness in Classical Mechanics, International Journal of Theoretical Physics, Vol. 30, \#8, August, pp.1041-73.

[19] da Costa, Newton. C. A \& Francisco Doria (1991), Classical Physics and Penrose's Thesis, Foundations of Physics Letters, Vol. 4, \#4, August, pp.363-73.

[20] Courant, Richard \& David Hilbert, (1962), Methods of Mathematical Physics, Vol I \& II, John Wiley \& Sons, New York.

[21] Davis, Martin (1958), Computability and Unsolvability, McGrawHill, New York.

[22] Dewdney, Alexander. K (1984), A Computer Trap for the Busy Beaver, the Hardest-Working Turing Machine, Scientific American, Vol. 251, No.2, August, pp.10-17.

[23] Dummett, Michael (1977), Elements of Intuitionism, Clarendon Press, Oxford.

[24] Estep, Donald, (2002), Practical Analysis in One Variable, SpringerVerlag, New York \& Berlin.

[25] Feferman, Solomon, (1999), Does Mathematics Need new Axioms?, The American Mathematical Monthly, Vol. 106, \#2, february, pp.99-111.

[26] Fellows, Michael. R \& Michael A. Langston, Nonconstructive Tools for Proving Polynomial-Time Decidability, Journal of the Association for Computing Machinery, Vol. 35, \#3, July, pp.727-39.

[27] Feynman, Richard P \& A. R. Hibbs (1965), Quantum Mechanics and Path Integrals, McGraw-Hill Book Company, New York. 
[28] Franzén, Torkel, (2005), Gödel's Theorem: An Incomplete Guide to its Use and Abuse, A K Peters, Wellesley, Massachusetts.

[29] Gödel, Kurt, (1931), Über formal unentscheidbare Sätze der Principia Mathematica und verwandter Systeme, I, Monatshefte für Mathematik und Physik, Vol. 38, \#1, December, pp.173-198.

[30] Gödel, Kurt, (1986), Collected Works, Vol. 1: Publications, 19291936, edited by Solomon Feferman, et.al, Oxford University Press, Oxford.

[31] Goodstein, Rebecca (2005): Incompleteness: The Proof and Paradox of Kurt Gödel, W.W. Norton and Company, Inc., New York.

[32] Greenleaf, Newcomb (1991), Algorithmic Languages and the Computability of Functions, in: The Mathematical Revolution Inspired by Computing, pp.221-32, edited by, J.H.Johnson \& M J Loomes, Clarendon Press, Oxford.

[33] Hirsch, Morris. W (1963), A Proof of the Non-Retractability of a Cell Onto its Boundary, Proceedings of the American Mathematical Society, Vol. 14, pp.364-5.

[34] Howie, John. M, (2001), Real Analysis, Springer-Verlag, London \& Berlin.

[35] Ince, E. L, (1944), Ordinary Differential Equations, Dover Pulbications, New York.

[36] Ishihara, Hajime, (1989), On the Constructive Hahn-Banach Theorem, Bulletin of the London Mathematical Society, Vol. 21, pp.79-81.

[37] Jones, Neil. D, (1997), Computability and Complexity: From a Programming Perspective, The MIT Press, Cambridge, Massachusetts.

[38] Joshi, Kapil D (1999), Mistake in Hirsch's Proof of the Brouwer Fixed Point Theorem, Proceedings of the American Mathematical Society, Vol. 128, \#5, pp.1523-5.

[39] Kamo, Hiroyasu, (2001), Effective Contraction Theorem and its Application, pp.88-100, in: Computability and Complexity in Analysis, edited by Jens Blanck, Vasco Brattka and Peter Hertling, Springer-Verlag, Berling \& Heidelberg.

[40] Kannai, Yakar, (1981), An Elementary Proof of the No-Retraction Theorem, American Mathematical Monthly, Vol. 88, \#4, April, pp.264-8.

[41] Kelley, John L, (1985), Recollections, in: Errett Bishop - Reflections on Him and His Research, pp.51-55, edited by Murray Rosenblatt, Contemporary Mathematics, Vol. 39, American Mathematical Society, Providence, Rhode Island. 
[42] Keynes, John Maynard, (1936), The General Theory of Employment, Interest and Money, Macmillan and Co., Limited, St. Martin's Street, London.

[43] Kleene, Stephen. C, (1952), Introduction to Metamathematics, D. Van Nostrand Company, Inc., Princeton, New Jersey.

[44] Kleene, Stephen. C, (1967), Mathematical Logic, John Wiley \& Sons, Inc., New York.

[45] Knuth, Donald. E, (1985), Algorithmic Thinking and Mathematical Thinking, American Mathematical Monthly, Vol. 92, \#3, March, pp.17081.

[46] Körner, T. W, (2003), A Companion to Analysis: A Second First and First Second Course in Analysis, American Mathematical Society, Providence, Rhode Island.

[47] Kragh, Helge, (1990), Dirac: A Scientific Biography, Cambridge University Press, Cambridge.

[48] Lin, Shin \& Tibor Rado (1965), Computer Studies of Turing Machine Problems, Journal of the Association for Computing Machinery, Vol. 12, No.2, April; pp.196-212.

[49] Ljunqvist, Lars and Thomas. J Sargent (2004), Recursive Macroeconomic Theory (Second Edition), The MIT Press, Cambridge, Massachusetts.

[50] Lucas, Robert. E, Jr., Nancy L. Stokey with Edward C. Prescott, (1989), Recursive Methods in Economic Dynamics, Harvard University Press, Cambridge, Massachusetts.

[51] Maitland Wright, J. D., (1977), Functional Analysis for the Practical Man, Functional Analysis: Surveys and Recent Results, edited by KlausDieter Bierstedt and Benno Fuchssteiner, North-Holland Publishing Company, Amsterdam \& New York.

[52] Mandelkern, Mark, (1985), Constructive Mathematics, Mathematics Magazine, Vol. 58, \#5, November, pp.272-80.

[53] Manin, Yu. I, (2002), Georg Cantor and His Heritage, arXiv:math/0209244v1 [math.AG], pp.12.

[54] Mar, Gary (2007), Review of [28], Mathematical Intelligencer, Vol. 20, \#3, Spring, pp.66-70.

[55] Marsden, Jerrold. E., \& Michael J. Hoffman, (1993), Elementary Classical Analysis (Second Edition), W.H. Freeman and Company, New York. 
[56] Mines, Ray, (1988), Review of Constructive Analysis by Errett Bishop and Douglas Bridges, American Mathematical Monthly, Vol. 95, \#2, February, pp.159-63.

[57] Moore, Gregory, H, (1982), Zermelo's Axiom of Choice: Its Origins, Development, and Influence, Springer-Verlag, New York \& Heidelberg.

[58] Moore, Ramon. E, (1966), Interval Analysis, Prentice-Hall, Englewood Cliffs, New Jersey.

[59] Moret, Bernard. M (1998): The Theory of Computation, AddisonWesley, Reading, Massachusetts.

[60] Moschovakis, Yiannis, N, (2001), What Is an Algorithm?, in: Mathematics Unlimited - 2001 and Beyond, pp.919-936, Springer-Verlag, Berlin and Heidelberg.

[61] Mount, Kenneth. R \& Stanley Reiter (2002), Computation and Complexity in Economic Organization, Cambridge University Press, Cambridge.

[62] Narici, Lawrence \& Edward Beckenstein, (1997), The Hahn-Banach Theorem, Topology and its Applications, Vol. 77, pp. 193-211.

[63] Nerode, Anil, George Metakides and Robert Constable, (1985), Recursive Limits on the Hahn-Banach Theorem, in: Errett Bishop - Reflections on Him and His Research, pp.85-91, edited by Murray Rosenblatt, Contemporary Mathematics, Vol. 39, American Mathematical Society, Providence, Rhode Island.

[64] Ok, Efe. A (2007), Real Analysis with Economic Applications, Princeton University Press, Princeton and London.

[65] Pawlikowski, Janusz, (1991), The Hahn-Banach Theorem Implies the Banach-Tarski Paradox, Fundamenta Mathematicae, Vol. 138, pp.2122.

[66] Peano, Giuseppe, 1890, Démonstration de l'intégrabilité des équations différentielles ordinaires, Mathematische Annalen, Vol. 37, pp.181-228

[67] Peano, Giiuseppe, 1973, Selected Works of Giuseppe Peano, Translated and Edited by Hubert C. Kennedy, George Allen \&Unwin, London.

[68] Post, Emil, (1944), Recursively Enumerable Sets of Positive Integers and their Decision Problems, Bulletin of the American Mathematical Society, Vol. 50, pp.284-316.

[69] Protter, M. H \& C. B. Morrey, (1977), A First Course in Real Analysis, Springer-Verlag, New York and Heidelberg. 
[70] Quine, Willard Van Orman, (1951), Mathematical Logic (Revised Edition), Harvard University Press, Cambridge, Massachusetts.

[71] Ramsey, Frank Plumpton (1926), The Foundations of Mathematics, Proceedings of the London Mathematical Society, Series 2, Vol.25, pp.338-84.

[72] Richman, Fred (1999), Existence Proofs, American Mathematical Monthly, Vol. 106, \#4, April, pp.303-8.

[73] Rado, Tibor (1962), On Non-Computable Functions, The Bell System Technical Journal, May, pp.877-884.

[74] Rogers, Hartley, Jr., (1967): Theory of Recursive Functions and Effective Computability, McGraw-Hill, New York.

[75] Rosser, J. Barkley (1936), Extensions of Some Theorems of Gödel and Church, Journal of Symbolic Logic, Vol. 1, \# 3, September, pp.87-91.

[76] Rosser, J. Barkley, (1938), The nth Prime is Greater than $n \log (n)$, Proceedings of the London Mathematical Society, Vol. 45, pp.2144.

[77] Rosser, J. Barkley, (1953): Logic for Mathematicians, McGraw-Hill, New York.

[78] Rosser, J. Barkley, (1969): Simplified Independence Proofs: Boolean Valued Models of Set Theory, Academic Press, New York and London

[79] Rosser, J. Barkley \& Carl de Boor, (1979), Pocket Calculator Supplement For Calculus, Addison-Wesley Publishing Company, Reading, Massachusetts.

[80] Rota, Gian-Carlo, (1986), In Memoriam of Stan Ulam: The Barrier of Meaning, Physica 22D, pp.1-3

[81] Russell, Bertrand (1908), Mathematical Logic as Based on the Theory of Types, American Journal of Mathematics, Vol.30, pp.222-62.

[82] Scarf, Herbert E (1973), The Computation of Economic Equilibria (with the collaboration of Terje Hansen), Yale University Press, New Haven and London.

[83] Scarf, Herbert E (1982), The Computation of Equilibrium Prices: An Exposition, Chapter 21, pp.1007-1061, in: Handbook of Mathematical Economics, Volume II, edited by Kenneth J. Arrow and Michael D. Intrilligator, North-Holland Publishing Company, Amsterdam.

[84] Schechter, Eric, (1997), Handbook of Analysis and Its Foundations, Academic Press, San Diego. 
[85] Schechter, Eric, (2001), Constructivism is Difficult, American Mathematical Monthly, Vol. 108, \#1, January, pp.50-54.

[86] Schwartz, Jacob. T., (1986), The Pernicious Influence of Mathematics on Science, Chapter 3, pp.19-25, in Discrete Thoughts by Marc kac, Gian-Carlo Rota and Jacob T. Schwartz, Borkhäuser, Boston \& Basel.

[87] Schwartz, Laurent, (2001), A Mathematician Grapples with His Century,Birkhäuser Verlag, Basel.

[88] Segre, Gavriel, (2007), The Mathematical Role of (Commutative and Noncommutative) infinitesimal random walks over (Commutative and noncommutative) Riemannian Manifolds in Quantum Physics, downloaded from: http://arxiv.org/abs/math-ph/0703011v4.

[89] Shoven, John B and John Whalley (1992), Applying General Equilibrium, Cambridge University Press, Cambridge.

[90] Specker, Ernst, (1949), Nicht konstruktiv beweisbare Sätze der Analysis, The Journal of Symbolic Logic, Vol. 14, \#3, pp.145-158.

[91] Sraffa, Piero, (1960), Production of Commodities by Means of Commodities: A Prelude to a Critique of Economic Theory, Cambridge University Press, Cambridge.

[92] Starr, Ross M (1977), General Equilibrium Theory: An Introduction, Cambridge University Press, Cambridge

[93] Stewart, Ian, (1991), Deciding the Undecidable, Nature, Vol. 352, 22 August, pp.664-5.

[94] Takagi, Teiji, (1903), A Simple Example of a Continuous Function without Derivative, Proceedings of the Physico-Mathematical Society of Japan, Vol. II-1, pp.176-7.

[95] Tasić, Vladimir, (2001), Mathematics and the Roots of Postmodern Thought, Oxford University Press, Oxford.

[96] Tompkins, Charles B (1964), Sperner's Lemma and Some Extensions, Chapter 15, pp. 416-55, in: Applied Combinatorial Mathematics edited by E.F. Beckenbach, John Wiley \& Sons. Ltd., New York.

[97] Troelstra, Anne. S, (1996), History of Constructivism in the 20th Century, downloaded from: http://turing.wins.uva.nl/ anne/ .

[98] Troelstra, Anne. S (undated), Constructivism and Proof Theory, downloaded from: staff.science.uva.nl/ ${ }^{\sim}$ anne/eolss.pdf.

[99] Turing, Alan, M, (1936-7), On Computable Numbers, with an Application to the Entscheidungsproblem, Proceedings of the Lodon Mathematical Society, Vol. 42, pp.230-65, Correction, Vol. 43, pp.544-6. 
[100] Uzawa, Hirofumi (1962), Walras' Existence Theorem and Brouwer's Fixed Point Theorem, The Economic Studies Quarterly, Vol. 8, No. 1, pp.59-62.

[101] Velupillai, K. Vela, (2007), Sraffa's Mathematical Economics: A Constructive Interpretation, forthcoming in The Journal of Economic Methodology (March or June, 2008).

[102] Wagner, K, (1937), Über eine Eigenschaft der Ebenen Complexe, Mathematische Annalen, Vol. 114, \#1, December, pp.570-90.

[103] Weihrauch, Klaus (2000), Computable Analysis: An Introduction, Springer-Verlag,Berlin \& Heidelberg.

[104] Wittgenstein, Ludwig, (1974), Philosophical Grammar, (edited by Rush Rhees \& translated by Anthony Kenny), Basil Blackwell, Oxford.

[105] Wolf, Robert, S, (2005), A Tour Through Mathematical Logic, The Carus Mathematical Monographs, \#30, The Mathematical Association of America, Washington, DC.

[106] Yourgrau, Palle (2005), A World Without Time: The Forgotten Legacy of Gödel and Einstein, Allen Lane, London.

[107] Zambelli, Stefano (2004), Production of Ideas by Means of Ideas, Metroeconomica, Vo. 55, \# 2\&3, May/September, pp.155-179.

[108] Zambelli, Stefano, (2005), Computable Knowledge and Undecidability: A Turing Machine Metaphor Applied to Endogenous Growth Models, in: Computability, Complexity and Constructivity in Economic Analysis, edited by K. Vela Velupillai, Blacwell Publishing, Oxford. 
Elenco dei papers del Dipartimento di Economia

2000.1 A two-sector model of the effects of wage compression on unemployment and industry distribution of employment, by Luigi Bonatti

2000.2 From Kuwait to Kosovo: What have we learned? Reflections on globalization and peace, by Roberto Tamborini

2000.3 Metodo e valutazione in economia. Dall'apriorismo a Friedman, by Matteo Motterlini

2000.4 Under tertiarisation and unemployment. by Maurizio Pugno

2001.1 Growth and Monetary Rules in a Model with Competitive Labor Markets, by Luigi Bonatti.

2001.2 Profit Versus Non-Profit Firms in the Service Sector: an Analysis of the Employment and Welfare Implications, by Luigi Bonatti, Carlo Borzaga and Luigi Mittone.

2001.3 Statistical Economic Approach to Mixed Stock-Flows Dynamic Models in Macroeconomics, by Bernardo Maggi and Giuseppe Espa.

2001.4 The monetary transmission mechanism in Italy: The credit channel and a missing ring, by Riccardo Fiorentini and Roberto Tamborini.

2001.5 Vat evasion: an experimental approach, by Luigi Mittone

2001.6 Decomposability and Modularity of Economic Interactions, by Luigi Marengo, Corrado Pasquali and Marco Valente.

2001.7 Unbalanced Growth and Women's Homework, by Maurizio Pugno

2002.1 The Underground Economy and the Underdevelopment Trap, by Maria Rosaria Carillo and Maurizio Pugno.

2002.2 Interregional Income Redistribution and Convergence in a Model with Perfect Capital Mobility and Unionized Labor Markets, by Luigi Bonatti.

2002.3 Firms' bankruptcy and turnover in a macroeconomy, by Marco Bee, Giuseppe Espa and Roberto Tamborini.

2002.4 One "monetary giant" with many "fiscal dwarfs": the efficiency of macroeconomic stabilization policies in the European Monetary Union, by Roberto Tamborini.

2002.5 The Boom that never was? Latin American Loans in London 18221825, by Giorgio Fodor. 
2002.6 L'economia senza banditore di Axel Leijonhufoud: le 'forze oscure del tempo e dell'ignoranza' e la complessità del coordinamento, by Elisabetta De Antoni.

2002.7 Why is Trade between the European Union and the Transition Economies Vertical?, by Hubert Gabrisch and Maria Luigia Segnana.

2003.1 The service paradox and endogenous economic gorwth, by Maurizio Pugno.

2003.2 Mappe di probabilità di sito archeologico: un passo avanti, di Giuseppe Espa, Roberto Benedetti, Anna De Meo e Salvatore Espa.

(Probability maps of archaeological site location: one step beyond, by Giuseppe Espa, Roberto Benedetti, Anna De Meo and Salvatore Espa).

2003.3 The Long Swings in Economic Understianding, by Axel Leijonhufvud.

2003.4 Dinamica strutturale e occupazione nei servizi, di Giulia Felice.

2003.5 The Desirable Organizational Structure for Evolutionary Firms in Static Landscapes, by Nicolás Garrido.

2003.6 The Financial Markets and Wealth Effects on Consumption An Experimental Analysis, by Matteo Ploner.

2003.7 Essays on Computable Economics, Methodology and the Philosophy of Science, by Kumaraswamy Velupillai.

2003.8 Economics and the Complexity Vision: Chimerical Partners or Elysian Adventurers?, by Kumaraswamy Velupillai.

2003.9 Contratto d'area cooperativo contro il rischio sistemico di produzione in agricoltura, di Luciano Pilati e Vasco Boatto.

2003.10 Il contratto della docenza universitaria. Un problema multi-tasking, di Roberto Tamborini.

2004.1 Razionalità e motivazioni affettive: nuove idee dalla neurobiologia e psichiatria per la teoria economica? di Maurizio Pugno.

(Rationality and affective motivations: new ideas from neurobiology and psychiatry for economic theory? by Maurizio Pugno.

2004.2 The economic consequences of Mr. G. W. Bush's foreign policy. Can th US afford it? by Roberto Tamborini

2004.3 Fighting Poverty as a Worldwide Goal by Rubens Ricupero

2004.4 Commodity Prices and Debt Sustainability by Christopher L. Gilbert and Alexandra Tabova 
2004.5 A Primer on the Tools and Concepts of Computable Economics by K. Vela Velupillai

2004.6 The Unreasonable Ineffectiveness of Mathematics in Economics by Vela K. Velupillai

2004.7 Hicksian Visions and Vignettes on (Non-Linear) Trade Cycle Theories by Vela K. Velupillai

2004.8 Trade, inequality and pro-poor growth: Two perspectives, one message? By Gabriella Berloffa and Maria Luigia Segnana

2004.9 Worker involvement in entrepreneurial nonprofit organizations. Toward a new assessment of workers? Perceived satisfaction and fairness by Carlo Borzaga and Ermanno Tortia.

2004.10 A Social Contract Account for CSR as Extended Model of Corporate Governance (Part I): Rational Bargaining and Justification by Lorenzo Sacconi

2004.11 A Social Contract Account for CSR as Extended Model of Corporate Governance (Part II): Compliance, Reputation and Reciprocity by Lorenzo Sacconi

2004.12 A Fuzzy Logic and Default Reasoning Model of Social Norm and Equilibrium Selection in Games under Unforeseen Contingencies by Lorenzo Sacconi and Stefano Moretti

2004.13 The Constitution of the Not-For-Profit Organisation: Reciprocal Conformity to Morality by Gianluca Grimalda and Lorenzo Sacconi

2005.1 The happiness paradox: a formal explanation from psycho-economics by Maurizio Pugno

2005.2 Euro Bonds: in Search of Financial Spillovers by Stefano Schiavo

2005.3 On Maximum Likelihood Estimation of Operational Loss Distributions by Marco Bee

2005.4 An enclave-led model growth: the structural problem of informality persistence in Latin America by Mario Cimoli, Annalisa Primi and Maurizio Pugno

2005.5 A tree-based approach to forming strata in multipurpose business surveys, Roberto Benedetti, Giuseppe Espa and Giovanni Lafratta.

2005.6 Price Discovery in the Aluminium Market by Isabel FiguerolaFerretti and Christopher L. Gilbert. 
2005.7 How is Futures Trading Affected by the Move to a Computerized Trading System? Lessons from the LIFFE FTSE 100 Contract by Christopher L. Gilbert and Herbert A. Rijken.

2005.8 Can We Link Concessional Debt Service to Commodity Prices? By Christopher L. Gilbert and Alexandra Tabova

2005.9 On the feasibility and desirability of GDP-indexed concessional lending by Alexandra Tabova.

2005.10 Un modello finanziario di breve periodo per il settore statale italiano: l'analisi relativa al contesto pre-unione monetaria by Bernardo Maggi e Giuseppe Espa.

2005.11 Why does money matter? A structural analysis of monetary policy, credit and aggregate supply effects in Italy, Giuliana Passamani and Roberto Tamborini.

2005.12 Conformity and Reciprocity in the "Exclusion Game": an Experimental Investigation by Lorenzo Sacconi and Marco Faillo.

2005.13 The Foundations of Computable General Equilibrium Theory, by K. Vela Velupillai.

2005.14 The Impossibility of an Effective Theory of Policy in a Complex Economy, by K. Vela Velupillai.

2005.15 Morishima's Nonlinear Model of the Cycle: Simplifications and Generalizations, by K. Vela Velupillai.

2005.16 Using and Producing Ideas in Computable Endogenous Growth, by K. Vela Velupillai.

2005.17 From Planning to Mature: on the Determinants of Open Source Take Off by Stefano Comino, Fabio M. Manenti and Maria Laura Parisi.

2005.18 Capabilities, the self, and well-being: a research in psychoeconomics, by Maurizio Pugno.

2005.19 Fiscal and monetary policy, unfortunate events, and the SGP arithmetics. Evidence from a growth-gap model, by Edoardo Gaffeo, Giuliana Passamani and Roberto Tamborini

2005.20 Semiparametric Evidence on the Long-Run Effects of Inflation on Growth, by Andrea Vaona and Stefano Schiavo.

2006.1 On the role of public policies supporting Free/Open Source Software. An European perspective, by Stefano Comino, Fabio M. Manenti and Alessandro Rossi. 
2006.2 Back to Wicksell? In search of the foundations of practical monetary policy, by Roberto Tamborini

2006.3 The uses of the past, by Axel Leijonhufvud

2006.4 Worker Satisfaction and Perceived Fairness: Result of a Survey in Public, and Non-profit Organizations, by Ermanno Tortia

2006.5 Value Chain Analysis and Market Power in Commodity Processing with Application to the Cocoa and Coffee Sectors, by Christopher L. Gilbert

2006.6 Macroeconomic Fluctuations and the Firms' Rate of Growth Distribution: Evidence from UK and US Quoted Companies, by Emiliano Santoro

2006.7 Heterogeneity and Learning in Inflation Expectation Formation: An Empirical Assessment, by Damjan Pfajfar and Emiliano Santoro

2006.8 Good Law \& Economics needs suitable microeconomic models: the case against the application of standard agency models: the case against the application of standard agency models to the professions, by Lorenzo Sacconi

2006.9 Monetary policy through the "credit-cost channel". Italy and Germany, by Giuliana Passamani and Roberto Tamborini

2007.1 The Asymptotic Loss Distribution in a Fat-Tailed Factor Model of Portfolio Credit Risk, by Marco Bee

2007.2 Sraffa?s Mathematical Economics - A Constructive Interpretation, by Kumaraswamy Velupillai

2007.3 Variations on the Theme of Conning in Mathematical Economics, by Kumaraswamy Velupillai

2007.4 Norm Compliance: the Contribution of Behavioral Economics Models, by Marco Faillo and Lorenzo Sacconi

2007.5 A class of spatial econometric methods in the empirical analysis of clusters of firms in the space, by Giuseppe Arbia, Giuseppe Espa e Danny Quah.

2007.6 Rescuing the LM (and the money market) in a modern Macro course, by Roberto Tamborini.

2007.7 Family, Partnerships, and Network: Reflections on the Strategies of the Salvadori Firm of Trento, by Cinzia Lorandini.

2007.8 I Verleger serici trentino-tirolesi nei rapporti tra Nord e Sud: un approccio prosopografico, by Cinzia Lorandini. 
2007.9 A Framework for Cut-off Sampling in Business Survey Design, by Marco Bee, Roberto Benedetti e Giuseppe Espa

2007.10 Spatial Models for Flood Risk Assessment, by Marco Bee, Roberto Benedetti e Giuseppe Espa

2007.11 Inequality across cohorts of households:evidence from Italy, by Gabriella Berloffa and Paola Villa

2007.12 Cultural Relativism and Ideological Policy Makers in a Dynamic Model with Endogenous Preferences, by Luigi Bonatti

2007.13 Optimal Public Policy and Endogenous Preferences: an Application to an Economy with For-Profit and Non-Profit, by Luigi Bonatti

2007.14 Breaking the Stability Pact: Was it Predictable?, by Luigi Bonatti and Annalisa Cristini.

2007.15 Home Production, Labor Taxation and Trade Account, by Luigi Bonatti.

2007.16 The Interaction Between the Central Bank and a Monopoly Union Revisited: Does Greater Uncertainty about Monetary Policy Reduce Average Inflation?, by Luigi Bonatti.

2007.17 Complementary Research Strategies, First-Mover Advantage and the Inefficiency of Patents, by Luigi Bonatti.

2007.18 DualLicensing in Open Source Markets, by Stefano Comino and Fabio M. Manenti.

2007.19 Evolution of Preferences and Cross-Country Differences in Time Devoted to Market Work, by Luigi Bonatti.

2007.20 Aggregation of Regional Economic Time Series with Different Spatial Correlation Structures, by Giuseppe Arbia, Marco Bee and Giuseppe Espa.

2007.21 The Sustainable Enterprise. The multi-fiduciary perspective to the EU Sustainability Strategy, by Giuseppe Danese.

2007.22 Taming the Incomputable, Reconstructing the Nonconstructive and Deciding the Undecidable in Mathematical Economics, by K. Vela Velupillai. 
PUBBLICAZIONE REGISTRATA PRESSO IL TRIBUNALE DI TRENTO 